\title{
A LIMIT THEOREM FOR SHIFTED SCHUR MEASURES
}

\author{
CRAIG A. TRACY and HAROLD WIDOM
}

\begin{abstract}
To each partition $\lambda=\left(\lambda_{1}, \lambda_{2}, \ldots\right)$ with distinct parts we assign the probability $Q_{\lambda}(x) P_{\lambda}(y) / Z$, where $Q_{\lambda}$ and $P_{\lambda}$ are the Schur $Q$-functions and $Z$ is a normalization constant. This measure, which we call the shifted Schur measure, is analogous to the much-studied Schur measure. For the specialization of the first $m$ coordinates of $x$ and the first $n$ coordinates of y equal to $\alpha(0<\alpha<1)$ and the rest equal to zero, we derive a limit law for $\lambda_{1}$ as $m, n \rightarrow \infty$ with $\tau=m / n$ fixed. For the Schur measure, the $\alpha$-specialization limit law was derived by Johansson [J1]. Our main result implies that the two limit laws are identical.
\end{abstract}

\section{Contents}

1. Introduction ... . . . . . . . . . . . . . . . . . . . . . 172

2. Schur $Q$-functions . . . . . . . . . . . . . . . . . . . . . . . . . . . . 174

3. Shifted RSK algorithm . . . . . . . . . . . . . . . . . . . . . . . . . . 178

4. A Gessel identity . . . . . . . . . . . . . . . . . . . . . . . . . . . . . 180

5. Shifted Schur measure . . . . . . . . . . . . . . . . . . . . . . . . . . 182

6. Proof of the main theorem . . . . . . . . . . . . . . . . . . . . . 184

6.1. An operator formulation . . . . . . . . . . . . . . . . . . . . . . 184

6.2. Calculation of $(I+\mathscr{K})^{-1}$. . . . . . . . . . . . . . . . . . . . . 188

6.3. Specialization .. . . . . . . . . . . . . . . . . . . 192

6.4. Scaling . . . . . . . . . . . . . . . . . . . . . . 194

6.5. Recapitulation .. . . . . . . . . . . . . . . . . . . 204

6.6. Computation of $\sigma^{\prime \prime \prime}\left(z_{0}\right)$. . . . . . . . . . . . . . . . . . . . . . 204

7. Poisson limit of the shifted Schur measure . . . . . . . . . . . . . . . 205

References ......................... 206

DUKE MATHEMATICAL JOURNAL

Vol. 123, No. 1, (C) 2004

Received 20 November 2002. Revision received 16 June 2003.

2000 Mathematics Subject Classification. Primary 60F05; Secondary 05E05, 05E10, 33E17, 47B35.

Authors' work supported by National Science Foundation grant numbers DMS-9802122 and DMS-9732687 and by the Mathematisches Forschungsinstitut Oberwolfach under the Research in Pairs program. 


\section{Introduction}

The Schur measure (see [O2]) assigns to each partition $\lambda=\left(\lambda_{1}, \lambda_{2}, \ldots\right)$ the weight

$$
s_{\lambda}(x) s_{\lambda}(y)
$$

where $s_{\lambda}$ are the Schur functions (see, e.g., $[\mathrm{M}],[\mathrm{St}]$ ). Thus

$$
\sum_{\substack{\lambda \in \mathscr{P} \\ \lambda_{1} \leq h}} s_{\lambda}(x) s_{\lambda}(y)
$$

is the (unnormalized) probability that $\lambda_{1}$, the number of boxes in the first row of the associated Young diagram, is less than or equal to $h$. Here $\mathscr{P}$ denotes the set of all partitions. The normalization constant, $Z$, is determined from the $h \rightarrow \infty$ limit

$$
Z:=\sum_{\lambda \in \mathscr{P}} s_{\lambda}(x) s_{\lambda}(y)=\prod_{i, j} \frac{1}{1-x_{i} y_{j}},
$$

where the last equality is the Cauchy identity for Schur functions. A theorem of Gessel [Ge] expresses the partition sum (1.1) as an $h \times h$ Toeplitz determinant $D_{h}(\varphi)$. It follows from this that the normalization constant is also given by

$$
Z=\lim _{h \rightarrow \infty} D_{h}(\varphi) .
$$

This limit can be explicitly computed by an application of the strong Szegö limit theorem (see, e.g., [BS]), and thereby the Cauchy identity reappears.

The Toeplitz determinant, or the Fredholm determinant coming from the Borodin-Okounkov identity (see [BO], [BW]), is the starting point in the analysis of limit laws for $\lambda_{1}$. This analysis together with the Robinson-Schensted-Knuth (RSK) correspondence gives a new class of limit laws, first discovered in the context of random matrix theory (see [TW1], [TW2]), for a number of probability models. Indeed, the result of Baik, Deift, and Johansson [BDJ] for the limit law of the length, $\ell_{N}(\pi)$, of the longest increasing subsequence in a random permutation $\pi \in \mathscr{S}_{N}$ is the nowclassic example. Exponential specialization of the Gessel identity together with the RSK correspondence* shows that

$$
\sum_{N=0}^{\infty} \mathrm{P}\left(\ell_{N} \leq h\right) \frac{t^{N}}{N !}
$$

is an $h \times h$ Toeplitz determinant with symbol $\varphi(z)=e^{\sqrt{t}(z+1 / z)}$. An asymptotic ${ }^{*}$ RSK associates bijectively to each permutation $\pi$ a pair of standard Young tableaux $(P, Q)$ of the same shape $\lambda$ such that $\ell_{N}(\pi)=\lambda_{1}$ (see, e.g., [St]). 
analysis of this Toeplitz determinant (using the steepest descent method for RiemannHilbert problems; see [DZ]) shows that

$$
\lim _{n \rightarrow \infty} \mathrm{P}\left(\frac{\ell_{N}-2 \sqrt{N}}{N^{1 / 6}}<s\right)=F_{2}(s)
$$

where $F_{2}$ is the limiting distribution of the largest eigenvalue (suitably centered and normalized) in the Gaussian unitary ensemble (see [TW1]). Similar results hold for longest increasing subsequences in symmetrized random permutations (see [BR3], [BR2]) and random words (see [AM], [J2], [ITW], [TW3]), for height fluctuations in various growth models (see [BR1], [GTW1], [GTW2], [J1]), and for tiling problems (see [J3]), as well as for extensions to the other rows of the Young diagram (see [BOO], [J2], [O1]).

In the theory of symmetric functions, there are many important generalizations of Schur functions (see [M]). These generalizations satisfy Cauchy identities, and it is natural to inquire about more general Gessel identities. However, one quickly sees that without determinantal formulas of the type that exist for Schur functions (the Jacobi-Trudi identity), Gessel identities seem unlikely. Nevertheless, the question of possible limit laws for sums of type (1.1) remains interesting.

This paper initiates work in this direction. Instead of Schur functions, we work with Schur $Q$-functions that have Pfaffian representations. These functions, introduced by Schur in 1911 in his analysis of the projective representation of the symmetric group, now have a combinatorial theory that parallels the combinatorial theory of Schur functions. This theory, due to Sagan [S] and Worley [Wo] (see also [Ste], [HH]), is based on a shifted version of the RSK algorithm. Whereas the usual RSK algorithm associates bijectively to each $\mathbb{N}$-matrix $A$ a pair of semistandard Young tableaux, the shifted RSK algorithm associates bijectively to each $\mathbb{P}$-matrix* $A$ a pair of shifted Young tableaux. There is a notion of increasing paths, and the length of the maximal path, $L(A)$, equals the number of boxes in the first row of the shifted tableau. Thus it is natural to assign to each partition $\lambda$ into distinct parts, that is, a strict partition, the probability

$$
\mathrm{P}(\{\lambda\})=\frac{1}{Z} Q_{\lambda}(x) P_{\lambda}(y),
$$

where $Q_{\lambda}$ and $P_{\lambda}$ are the Schur $Q$-functions and $Z$ is a normalization constant. We call this measure the shifted Schur measure.

At first our analysis is for general parameters $x$ and $y$ appearing in the shifted Schur measure, and we find that there is indeed a Gessel identity. (It follows from the Ishikawa-Wakayama Pfaffian summation formula [IW].) Then we specialize the

\footnotetext{
*Informally, a $\mathbb{P}$-matrix is an $\mathbb{N}$-matrix where we allow the nonzero entries to be either marked or unmarked. Precise definitions are given below.
} 
measure by choosing the first $m$ coordinates of $x$ and the first $n$ coordinates of $y$ equal to $\alpha(0<\alpha<1)$ and the rest equal to zero. We call this $\alpha$-specialization and denote the resulting specialized shifted Schur measure by $\mathrm{P}_{\sigma}$, where $\sigma=(m, n, \alpha)$ denotes the parameters of the measure. Now, however, the matrix on the right side of the Gessel identity is not Toeplitz, and so the earlier analytical methods are not immediately available to us. Nevertheless, we do find that the distribution function for $L(A)=\lambda_{1}$ can be expressed in terms of the Fredholm determinant of an operator which is a perturbation of a direct sum of products of Hankel operators. In the end we can show that the trace norms of the perturbations tend to zero and are able to determine the asymptotics.

We asume* that $m / n=\tau$ is a constant satisfying $\alpha^{2}<\tau<\alpha^{-2}$. Our main result is the following.

\section{MAIN THEOREM}

Let $P_{\sigma}$ denote the $\alpha$-specialized shifted Schur measure with $\tau$ satisfying the stated restriction. Then there exist constants $c_{1}=c_{1}(\alpha, \tau)$ and $c_{2}=c_{2}(\alpha, \tau)$ such that

$$
\lim _{n \rightarrow \infty} P_{\sigma}\left(\frac{\lambda_{1}-c_{1} n}{c_{2} n^{1 / 3}}<s\right)=F_{2}(s)
$$

For $\tau=1$, the constants have a particularly simple form, namely,

$$
c_{1}(\alpha, 1)=\frac{4 \alpha}{1-\alpha^{2}} \quad \text { and } \quad c_{2}(\alpha, 1)=\frac{\left(2 \alpha\left(1+6 \alpha^{2}+\alpha^{4}\right)\right)^{1 / 3}}{1-\alpha^{2}} .
$$

Expressions for $c_{1}$ and $c_{2}$ in general are given in $\$ 6$. For the Schur measure, the corresponding $\alpha$-specialization limit law was derived by Johansson [J1]; as the main theorem shows, the two limit laws are identical. ${ }^{\dagger}$ The table of contents provides a description of the layout of this paper.

\section{Schur $Q$-functions}

This section and $\S 3$ summarize the properties of the Schur $Q$-functions and the shifted RSK algorithm which we need in this paper. The material is not new on our part. It is presented to establish the notation used in subsequent sections and as a convenience to the reader. A complete presentation can be found in the books by Macdonald [M] and Hoffman and Humphreys [HH], in the papers by Sagan [S] and Stembridge [Ste], and in the dissertation of Worley [Wo].

\footnotetext{
*The stated restriction on $\tau$ is very likely unnecessary for the validity of the final result. Some details of the proof would be different in the other cases, but we did not carry them out.

$\dagger$ We note that our $\alpha$ is related to Johansson's $q$ by $q=\alpha^{2}$. For the $\alpha$-specialized Schur measure, $c_{1}(\alpha, 1)=$ $2 \alpha /(1-\alpha)$ and $c_{2}(\alpha, 1)=\alpha^{1 / 3}(1+\alpha)^{1 / 3} /(1-\alpha)$.
} 
If $\lambda=\left(\lambda_{1}, \lambda_{2}, \ldots\right)$ is a partition of $n$, we denote this by $\lambda \vdash n$. The length of $\lambda$ is denoted by $\ell(\lambda)$. Let $\mathscr{P}_{n}$ denote the set of all partitions of $n$, and let $\mathscr{P}:=\bigcup_{n=0}^{\infty} \mathscr{P}_{n}$. ( $\mathscr{P}_{0}$ is the empty partition.) Introduce $\mathscr{D}_{n} \subset \mathscr{P}_{n}$, the set of partitions of $n$ into distinct parts. For example,

$$
\mathscr{D}_{6}=\{\{6\},\{5,1\},\{4,2\},\{3,2,1\}\} .
$$

Let $\mathscr{D}:=\bigcup_{n=0}^{\infty} \mathscr{D}_{n}$, the set of all partitions into distinct parts. If $\lambda \vdash n$ is a partition with distinct parts, we denote this by $\lambda \models n$ and call $\lambda$ a strict partition of $n$.

Associated to a strict partition $\lambda$ is a shifted shape $S(\lambda)$. One starts with the usual Young diagram $Y(\lambda)$ and for $i=1,2, \ldots, \ell(\lambda)$ simply indents the $i$ th row to the right by $i-1$ boxes. The result is $S(\lambda)$. We usually use $\lambda \in \mathscr{D}$ to denote both a strict partition and the shifted shape $S(\lambda)$.

We let $\mathbb{N}$ denote the set of positive integers, and

$$
\mathbb{P}=\left\{1^{\prime}, 1,2^{\prime}, 2,3^{\prime}, 3, \ldots\right\}
$$

with the ordering

$$
1^{\prime}<1<2^{\prime}<2<3^{\prime}<3<\cdots .
$$

We call the elements either marked or unmarked, depending on whether the element is primed or not. When we do not wish to distinguish a marked element $m^{\prime}$ from the unmarked element $m$, we write $m^{*}$. A (generalized) shifted Young tableau, $T$, is an assignment of elements of $\mathbb{P}$ to a shifted shape $\lambda$ having the following properties.

(T1) $T$ is weakly increasing across rows and down columns.

(T2) For each integer $m^{*}$, there is at most one $m^{\prime}$ in each row and at most one $m$ in each column of $T$. (Thus the marked elements are strictly increasing across rows of $T$ and the unmarked elements are strictly increasing down columns of T.)

An example of a shifted tableau of shape $(7,5,3,2,1)$ is

$$
\begin{array}{ccccccc}
1^{\prime} & 1 & 2^{\prime} & 2 & 2 & 5^{\prime} & 6 \\
& 2^{\prime} & 2 & 3^{\prime} & 4 & 5 & \\
& & 3^{\prime} & 4 & 5 & & \\
& & & 6 & 7^{\prime} & & \\
& & & & 7^{\prime} . & &
\end{array}
$$

To each shifted tableau $T$ we associate a monomial

$$
x^{T}=x_{1}^{a_{1}} x_{2}^{a_{2}} \cdots x_{m}^{a_{m}} \cdots,
$$

where $a_{m}$ is the number of times $m^{*}$ appears in $T$. Thus as far as the monomial is concerned, we do not distinguish between marked and unmarked elements. In the above example,

$$
x^{T}=x_{1}^{2} x_{2}^{5} x_{3}^{2} x_{4}^{2} x_{5}^{3} x_{6}^{2} x_{7}^{2}
$$


Let $\lambda$ be a strict partition of $n$. The Schur $Q$-function, the generating function of shifted tableaux of shape $\lambda$, is

$$
Q_{\lambda}(x):=\sum_{T} x^{T}
$$

where the sum runs over all shifted tableaux of shape $\lambda \models n$. The Schur $Q$-function is the analogue of the Schur function $s_{\lambda}$ when one replaces semistandard Young tableaux of shape $\lambda$ by shifted tableaux of shape $\lambda$. (Of course, here $\lambda$ must be a strict partition.) It is convenient to introduce the Schur $P$-function

$$
P_{\lambda}(x)=\frac{1}{2^{\ell(\lambda)}} Q_{\lambda}(x) .
$$

We remark that a shifted tableau $T$ of shifted shape $\lambda \models n$ is called standard if it has no marked elements and uses each unmarked letter $1,2, \ldots, n$ exactly once. Schur showed that the number of standard shifted tableaux of shape $\lambda \models n, \lambda=$ $\left(\lambda_{1}, \lambda_{2}, \ldots, \lambda_{\ell}\right)$, is

$$
f_{s}^{\lambda}=\frac{n !}{\lambda_{1} ! \lambda_{2} ! \cdots \lambda_{\ell} !} \prod_{1 \leq i<j \leq \ell} \frac{\lambda_{i}-\lambda_{j}}{\lambda_{i}+\lambda_{j}} .
$$

This should be compared with the number of standard Young tableaux

$$
f^{\lambda}=\frac{n !}{\ell_{1} ! \ell_{2} ! \cdots \ell_{k} !} \prod_{1 \leq i<j \leq k}\left(\ell_{i}-\ell_{j}\right),
$$

where $\lambda=\left(\lambda_{1}, \lambda_{2}, \ldots, \lambda_{k}\right)$ and $\ell_{j}=\lambda_{j}+k-j$. The number of semistandard Young tableaux of shape $\lambda$ which can be formed using the integers $1,2, \ldots, n$ is

$$
d^{\lambda}(n)=s_{\lambda}(\overbrace{1, \ldots, 1}^{n}, 0,0, \ldots) .
$$

Similarly, the number of shifted tableaux of shape $\lambda$ which can be formed using the integers $1^{*}, 2^{*}, \ldots, n^{*}$ is

$$
d_{s}^{\lambda}(n)=Q_{\lambda} \overbrace{1, \ldots, 1}^{n}, 0,0, \ldots) .
$$

This specialization of $Q_{\lambda}$ is important below.

The Schur $Q$-functions satisfy a Cauchy identity

$$
\sum_{\lambda \in \mathscr{D}} Q_{\lambda}(x) P_{\lambda}(y)=\prod_{i, j=1}^{\infty} \frac{1+x_{i} y_{j}}{1-x_{i} y_{j}}=Z .
$$


The right-hand side enumerates all matrices $A$ whose entries are chosen from $\mathbb{P} \cup\{0\}$ : the denominator counts matrices with entries in $\mathbb{N} \cup\{0\}$, while the numerator accounts for the primes. We call these matrices $\mathbb{P}$-matrices. The above product is frequently specialized to $x=\left(x_{1}, x_{2}, \ldots, x_{m}, 0, \ldots\right)$ and $y=\left(y_{1}, y_{2}, \ldots, y_{n}, 0, \ldots\right)$. We use the same symbol $Z$ to denote this specialization. It is clear from the context how to interpret $Z$.

Define symmetric functions $q_{k}$ by

$$
Q(t):=\prod_{i=1}^{\infty} \frac{1+t x_{i}}{1-t x_{i}}=\sum_{k=0}^{\infty} q_{k}(x) t^{k} .
$$

(When necessary to indicate the dependence upon $x$, we write $Q(t, x)$.) It follows from $Q(t) Q(-t)=1$ that

$$
q_{2 m}=\sum_{r=1}^{m-1}(-1)^{r-1} q_{r} q_{2 m-r}+\frac{1}{2}(-1)^{m} q_{m}^{2},
$$

which shows that $q_{2 m} \in \mathbb{Q}\left[q_{1}, q_{2}, \ldots, q_{2 m-1}\right]$ and hence by induction on $m$,

$$
q_{2 m} \in \mathbb{Q}\left[q_{1}, q_{3}, q_{5}, \ldots, q_{2 m-1}\right] .
$$

Denote by $\Gamma$ the subring of $\Lambda$ generated by the $q_{r}$,

$$
\Gamma=\mathbb{Z}\left[q_{1}, q_{3}, \ldots\right] .
$$

If $\lambda=\left(\lambda_{1}, \lambda_{2}, \ldots\right)$, we let

$$
q_{\lambda}:=q_{\lambda_{1}} q_{\lambda_{2}} \cdots
$$

It is known that the $q_{\lambda}$ with $\lambda$ strict form a $\mathbb{Z}$-basis of $\Gamma$.

We now give the classical definition of the Schur $Q$-function. (Of course, in this presentation it is a theorem.) If $\lambda$ is a strict partition of length at most $n$, then $Q_{\lambda}$ equals the coefficient of $t^{\lambda}:=t_{1}^{\lambda_{1}} t_{2}^{\lambda_{2}} \cdots$ in

$$
Q\left(t_{1}, t_{2}, \ldots, t_{n}\right)=\prod_{i=1}^{n} Q\left(t_{i}\right) \prod_{i<j} F\left(t_{i}^{-1} t_{j}\right),
$$

where

$$
F(y)=\frac{1-y}{1+y}=1+2 \sum_{r \geq 1}(-1)^{r} y^{r}
$$

and $Q$ is defined by (2.4); in particular, for $r>s$,

$$
Q_{(r, s)}=\left(\frac{1}{2 \pi \mathrm{i}}\right)^{2} \iint t_{1}^{-r-1} t_{2}^{-s-1} F\left(t_{2} / t_{1}\right) Q\left(t_{1}\right) Q\left(t_{2}\right) \mathrm{d} t_{1} \mathrm{~d} t_{2},
$$


where the contours could be chosen to be circles with $\left|t_{2}\right|<\left|t_{1}\right|$. $^{*}$

Here are some additional properties of Schur $Q$-functions.

(1) The $Q_{\lambda}, \lambda$ strict, form a $\mathbb{Z}$-basis of $\Gamma$.

(2) Using (2.5) and the expansion for $F$, we have for $r>s \geq 0$,

$$
Q_{(r, s)}=q_{r} q_{s}+2 \sum_{i=1}^{s}(-1)^{i} q_{r+i} q_{s-i} .
$$

For $r \leq s$, we define $Q_{(r, s)}=-Q_{(s, r)}$. Now let $\lambda$ be a strict partition that we write in the form $\lambda=\left(\lambda_{1}, \lambda_{2}, \ldots, \lambda_{2 n}\right)$, where $\lambda_{1}>\lambda_{2}>\cdots>\lambda_{2 n} \geq 0$. Define the $2 n \times 2 n$ antisymmetric matrix

$$
M_{\lambda}=\left(Q_{\left(\lambda_{i}, \lambda_{j}\right)}\right)
$$

then we have

$$
Q_{\lambda}=\operatorname{pf}\left(M_{\lambda}\right),
$$

where pf denotes the Pfaffian.

\section{Shifted RSK algorithm}

For later convenience, we use a nonstandard labeling of matrix $A$ : rows are numbered starting at the lower left-hand corner of $A$, and columns have the usual left-to-right labeling. To each $\mathbb{P}$-matrix $A$ we (bijectively) associate a biword $w_{A}$ as follows. For a fixed column index we scan the matrix for increasing values of the row index. If the $(i, j)$-entry is unmarked with value $a_{i j}$, we repeat the pair $\left(\begin{array}{c}j \\ i\end{array}\right) a_{i j}$ times in $w_{A}$. If the $(i, j)$-element is marked, the $i$ of the first pair $\left(\begin{array}{c}j \\ i\end{array}\right)$ appearing in $w_{A}$ is marked. For example, if

$$
A=\left(\begin{array}{ccc}
1 & 2 & 0 \\
1^{\prime} & 0 & 2^{\prime} \\
3^{\prime} & 0 & 1
\end{array}\right),
$$

then

$$
w_{A}=\left(\begin{array}{cccccccccc}
1 & 1 & 1 & 1 & 1 & 2 & 2 & 3 & 3 & 3 \\
1^{\prime} & 1 & 1 & 2^{\prime} & 3 & 3 & 3 & 1 & 2^{\prime} & 2
\end{array}\right) .
$$

A description of the shifted RSK algorithm is more involved than that of the usual RSK algorithm, though the general features remain the same. Namely, there is a row bumping (and column bumping) procedure that, when iterated on a sequence

*This requires that $x \in \ell^{1}$ and that the poles $x_{i}^{-1}$ lie outside the contours. Notice that if the $t_{1}$-contour were deformed to one inside the $t_{2}$-contour, then since $Q(t) Q(-t)=1$, the residue at the pole $t_{1}=-t_{2}$ crossed would be $2 t_{2}^{-r-s-1}$. The integral of this equals zero as long as $r$ and $s$ are not both zero. This shows that the contours can also be chosen so that $\left|t_{2}\right|>\left|t_{1}\right|$. Equivalently, the integral representation holds for $r<s$ as well. 
$\alpha$ whose elements are in $\mathbb{P}$, gives a shifted tableau $S$, the insertion tableau. (This is applied to the sequence in the bottom half of the biword $w_{A}$.) The top half of $w_{A}$ gives a recording tableau $T$. We now state the final result, referring the interested reader to either $[\mathrm{HH}]$ or $[\mathrm{S}]$.

\section{THEOREM (Sagan [S], Worley [Wo])}

There is a bijective correspondence between $\mathbb{P}$-matrices $A=\left(a_{i j}\right)$ and ordered pairs $(S, T)$ of shifted tableaux of the same shape, such that $T$ has no marked letters on its main diagonal. The correspondence has the property that $\sum_{i} a_{i j}^{*}$ is the number of entries $t$ of $T$ for which $t^{*}=j$, whereas $\sum_{j} a_{i j}^{*}$ is the number of entries $s$ of $S$ for which $s^{*}=i$. (Recall that the ${ }^{*}$ means we do not distinguish between a marked or unmarked form of an integer.)

We designate the matrix $S$, respectively, $T$, to be of type $s$, respectively, $t$.

An important property of the RSK algorithm is its relationship to increasing subsequences of maximal length in the biword $w_{A}$ (equivalently, increasing paths in the matrix $A$ of maximal weight). The shifted RSK algorithm of Sagan and Worley shares a similar property once the notion of an increasing subsequence is properly formulated. Let $\psi \in \mathbb{P}^{m}, \phi \in \mathbb{P}^{n}$, and denote by $\psi \sqcup \phi$ the concatenation $\left(\psi_{1}, \ldots, \psi_{m}, \phi_{1}, \ldots, \phi_{n}\right)$. Denote by $\operatorname{rev}(\phi)$ the reverse of $\phi, \operatorname{rev}(\phi)=$ $\left(\phi_{n}, \phi_{n-1}, \ldots, \phi_{1}\right)$. Given a sequence $\alpha$ from $\mathbb{P}$, an ascent pair $(\psi, \phi)$ for $\alpha$ is a pair of subsequences $\psi$ of $\operatorname{rev}(\alpha)$ and $\phi$ of $\alpha$ such that if $\psi \in \mathbb{P}^{m}$ and $\phi \in \mathbb{P}^{n}$, then

(1) $\quad \psi \sqcup \phi$ is weakly increasing with respect to the ordering of $\mathbb{P}$;

(2) for all $k \in \mathbb{N}$, at most one (unmarked) $k$ appears in $\psi$;

(3) for all $k \in \mathbb{N}$, at most one (marked) $k^{\prime}$ appears in $\phi$.

Thus the unmarked symbols are strictly increasing in $\psi$ and the marked symbols are strictly increasing in $\phi$. The length of $\psi \sqcup \phi$ is defined to be $m+n-1$. (Note that the length here is one less than the length defined in either [HH] or [S].) Let $\alpha \in \mathbb{P}^{n}$, and let $L(\alpha)$ denote the length of the longest ascent pair $(\psi, \phi)$ of $\alpha$. Then we have the following.

\section{THEOREM (Sagan [S], Worley [Wo])}

If $\alpha$ is a sequence from $\mathbb{P}$ and $T$ is the shifted tableau of shape $\left(\lambda_{1}, \lambda_{2}, \ldots, \lambda_{k}\right)$ resulting from the insertion of $\alpha$ (following the rules of the shifted RSK algorithm), then $L(\alpha)=\lambda_{1}$.

Here is an example of an increasing path displayed in the $\mathbb{P}$-matrix $A$. In this example, 
$L(A)=16:^{*}$

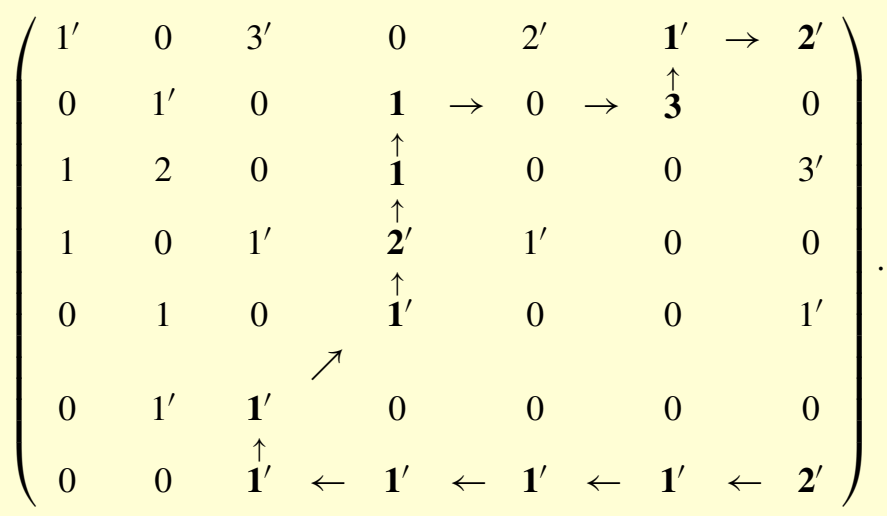

A quick way to compute this length is to apply a modified patience sorting algorithm (see $[\mathrm{AD}]$ ) to the lower row of the associated biword. (We leave this as an exercise for the interested reader.) Of course, another way is to apply the shifted RSK algorithm and count the number of boxes in the first row.

\section{A Gessel identity}

The Gessel identity [Ge] (see also [TW3]) states that the sum

$$
\sum_{\substack{\lambda \in \mathscr{P} \\ \lambda_{1} \leq h}} s_{\lambda}(x) s_{\lambda}(y)
$$

equals a certain $h \times h$ Toeplitz determinant. The proof begins by expressing the Schur functions $s_{\lambda}$ as determinants (the Jacobi-Trudi identity) and proceeds by recognizing this sum of products of determinants as the expansion of a single determinant of the product of two (nonsquare) matrices. (This expansion is called the Cauchy-Binet expansion.)

We are interested in sums of the form

$$
\sum_{\substack{\lambda \in \mathscr{D} \\ \lambda_{1} \leq h}} Q_{\lambda}(x) P_{\lambda}(y),
$$

where now, as we have seen, the $Q_{\lambda}$ and $P_{\lambda}$ are given by Pfaffians. What is needed is a Pfaffian version of the Cauchy-Binet formula. Fortunately, Ishikawa and Wakayama [IW] have such a formula (see also Stembridge [Ste]).

Introduce

$$
I_{r}^{h}=\left\{I=\left(i_{1}, \ldots, i_{r}\right): 1 \leq i_{1}<\cdots<i_{r} \leq h\right\},
$$

${ }^{*}$ Note, for example, that the path segment $\mathbf{1}^{\prime} \rightarrow \mathbf{2}^{\prime}$ in the upper right-hand corner contributes a weight of two, not three, since the marked elements are strictly increasing. 
and denote by $A_{J}$ the submatrix formed from $A$ by taking those rows and columns indexed by $J \in I_{r}^{h}$. Then the Pfaffian summation formula is the following.

THEOREM (Ishikawa and Wakayama [IW])

Let $A=\left(a_{i j}\right)_{0 \leq i, j \leq h}$ and $B=\left(b_{i j}\right)_{0 \leq i, j \leq h}$ be $(h+1) \times(h+1)$ skew-symmetric matrices, $h \in \mathbb{N}$. Then

$$
\begin{aligned}
\sum_{\substack{0 \leq r \leq h \\
r \text { even }}} \sum_{I \in I_{r}^{h}} \gamma^{|I|} \operatorname{pf}\left(A_{I}\right) \operatorname{pf}\left(B_{I}\right)+\sum_{\substack{0 \leq r \leq h \\
r \text { odd }}} \sum_{I \in I_{r}^{h}} \gamma^{|I|} \operatorname{pf}\left(A_{0 I}\right) \operatorname{pf}\left(B_{0 I}\right) \\
=(-1)^{h(h-1) / 2} \operatorname{pf}\left(\begin{array}{cc}
-A & I_{h+1} \\
-I_{h+1} & C
\end{array}\right),
\end{aligned}
$$

where $C=\left(C_{i j}\right)_{0 \leq i, j \leq h}$ is the $(h+1) \times(h+1)$ antisymmetric matrix

$$
C_{i j}= \begin{cases}\gamma^{j} b_{0 j} & \text { if } i=0, j \geq 1, \\ \gamma^{i} b_{i 0} & \text { if } i \geq 1, j=0, \\ \gamma^{i+j} b_{i j} & \text { if } i \geq 1, j \geq 1 .\end{cases}
$$

Here $|I|=\sum i_{k}$, and $A_{0 I}$, where $I=\left\{i_{1}, i_{2}, \ldots\right\}$, stands for $A_{J}$, where $J=$ $\left\{0, i_{1}, i_{2}, \ldots\right\}$.

Observe that $I_{r}^{h}$ is the set of partitions with exactly $r$ distinct parts such that the largest part is at most $h$. For any such partition, $r \leq h$. In (4.1) we break the sum into two sums - the first sum over distinct partitions with an even number of parts and the second sum over distinct partitions with an odd number of parts. Recalling the Pfaffian representation (2.7) of $Q_{\lambda}$, we note that if $\lambda$ has an odd number of parts, then we extend the partition by appending zero, giving us a vector of even length. Thus the sum appearing in the Pfaffian summation formula is (up to a reversal of labels) the sum over distinct partitions satisfying $\lambda_{1} \leq h$. From (2.7), we see that $A_{h}(x)$ is the $(h+1) \times(h+1)$ antisymmetric matrix

$$
\left(\begin{array}{cc}
0 & -q^{t} \\
q & \widehat{Q}_{h}(x)
\end{array}\right)
$$

Here $q$ is the $(h \times 1)$-matrix with elements $q_{r}(x)(r=1,2, \ldots, h), \widehat{Q}_{h}(x)$ is the $h \times h$ antisymmetric matrix with elements $Q_{(r, s)}(x)$, and $q^{t}$ denotes the transpose of $q$. (Recall that $Q_{(r, 0)}=q_{r}$.) The Pfaffian representation for $P_{\lambda}$ is obtained from the 
$Q_{\lambda}$ Pfaffian representation by inserting the factor $2^{-\ell(\lambda)}$. The matrix $B_{h}$ is

$$
\begin{aligned}
B_{h}(y) & =\left(\begin{array}{cc}
0 & -\frac{1}{2} q^{t} \\
\frac{1}{2} q & \frac{1}{4} \widehat{Q}_{h}(y)
\end{array}\right) \\
& =\left(\begin{array}{ll}
1 & 0 \\
0 & \frac{1}{2}
\end{array}\right) A_{h}(y)\left(\begin{array}{ll}
1 & 0 \\
0 & \frac{1}{2}
\end{array}\right) .
\end{aligned}
$$

Applying the summation formula then gives for $h \in \mathbb{N}$,

$$
\begin{aligned}
\sum_{\substack{\lambda \in \mathscr{D} \\
\lambda_{1} \leq h}} Q_{\lambda}(x) P_{\lambda}(y) & =\operatorname{pf}\left(\begin{array}{cc}
-A_{h}(x) & I \\
-I & B_{h}(y)
\end{array}\right) \\
& =\left(\operatorname{det}\left(I-A_{h}(x) B_{h}(y)\right)\right)^{1 / 2} .
\end{aligned}
$$

The \pm -factors are accounted for by the reversal of labels or, more simply, because we are computing probabilities.

Introducing the antisymmetric matrix

$$
K_{h}(x)=\left(\begin{array}{cc}
1 & 0 \\
0 & \frac{1}{\sqrt{2}}
\end{array}\right) A_{h}(x)\left(\begin{array}{cc}
1 & 0 \\
0 & \frac{1}{\sqrt{2}}
\end{array}\right)
$$

or, more explicitly,

$$
K_{h}(x)_{r s}= \begin{cases}-\frac{1}{\sqrt{2}} q_{s}(x), & r=0, s \geq 1, \\ \frac{1}{\sqrt{2}} q_{r}(x), & r \geq 1, s=0, \\ \frac{1}{2} Q_{(r, s)}(x), & r \geq 1, s \geq 1,\end{cases}
$$

we obtain our Gessel identity,

$$
\sum_{\substack{\lambda \in \mathscr{D} \\ \lambda_{1} \leq h}} Q_{\lambda}(x) P_{\lambda}(y)=\left(\operatorname{det}\left(I-K_{h}(x) K_{h}(y)\right)\right)^{1 / 2} .
$$

Observe that it follows from (4.3) and the Cauchy identity (2.3) that

$$
\lim _{h \rightarrow \infty} \operatorname{det}\left(I-K_{h}(x) K_{h}(y)\right)=Z^{2} .
$$

\section{Shifted Schur measure}

Let $\mathscr{P}_{m, n}$ denote the set of $\mathbb{P}$-matrices of size $m \times n$. For $A \in \mathscr{P}_{m, n}$, we recall that $L(A)$ denotes the length of the longest increasing path in $A$. Let $x=\left(x_{1}, x_{2}, \ldots\right)$ and $y=\left(y_{1}, y_{2}, \ldots\right)$ with $0 \leq x_{i}<1$ and $0 \leq y_{i}<1$. We assume that the matrix elements $a_{i j}$ are distributed independently with a geometric distribution with parameter $x_{i} y_{j}$. Specifically, for $k \geq 1$,

$$
\mathrm{P}\left(a_{i j}=k\right)=\mathrm{P}\left(a_{i j}=k^{\prime}\right)=\left(\frac{1-x_{i} y_{j}}{1+x_{i} y_{j}}\right)\left(x_{i} y_{j}\right)^{k}
$$


and

$$
\mathrm{P}\left(a_{i j}=0\right)=\frac{1-x_{i} y_{j}}{1+x_{i} y_{j}}
$$

We have, of course,

$$
\sum_{k^{*}=0}^{\infty} \mathrm{P}\left(a_{i j}=k^{*}\right)=\frac{1-x_{i} y_{j}}{1+x_{i} y_{j}}+2 \sum_{k \geq 1}\left(\frac{1-x_{i} y_{j}}{1+x_{i} y_{j}}\right)\left(x_{i} y_{j}\right)^{k}=1 .
$$

Let $\mathscr{P}_{m, n, s, t}\left(t \in \mathbb{N}^{m}, s \in \mathbb{N}^{n}\right)$ denote the set of $A \in \mathbb{P}_{m, n}$ satisfying, for $1 \leq i \leq$ $m$ and $1 \leq j \leq n$,

$$
\sum_{1 \leq j \leq n} a_{i j}^{*}=s_{i} \quad \text { and } \quad \sum_{1 \leq i \leq m} a_{i j}^{*}=t_{j} .
$$

Then for $A \in \mathscr{P}_{m, n, s, t}$ we have

$$
\mathrm{P}(\{A\})=\prod_{\substack{1 \leq i \leq m \\ 1 \leq j \leq n}}\left(\frac{1-x_{i} y_{j}}{1+x_{i} y_{j}}\right) x^{s} y^{t}=\frac{1}{Z} x^{s} y^{t} .
$$

Since the right-hand side does not depend upon the $A$ chosen in $\mathscr{P}_{m, n, s, t}$, the conditional probability

$$
\mathrm{P}\left(L \leq h \mid \sum_{j} a_{i j}^{*}=s_{i}, \sum_{i} a_{i j}^{*}=t_{j}\right)
$$

is uniform. Note that this uses both the independence and the geometric distribution of the random variables $a_{i j}$.

By the shifted RSK correspondence, to each $A \in \mathscr{P}_{m, n, s, t}$ we associate bijectively a pair $(S, T)$ of shifted tableaux of the same shape $\lambda \models N\left(N:=\sum_{i, j} a_{i j}^{*}\right)$ of types $s$ and $t$, respectively. The condition $L(A) \leq h$ becomes $\lambda_{1} \leq h$. Hence

$$
\begin{aligned}
\mathrm{P}_{m, n}(L \leq h) & =\sum_{A \in \mathscr{P}_{m, n} / \mathscr{P}_{m, n, s, t}} \mathrm{P}\left(\ell(A) \leq h \mid A \in \mathscr{P}_{m, n, s, t}\right) \mathrm{P}\left(A \in \mathscr{P}_{m, n, s, t}\right) \\
& =\sum_{A \in \mathscr{P}_{m, n} / \mathscr{P}_{m, n, s, t}} \frac{1}{\left|\mathscr{P}_{m, n, s, t}\right|} \frac{1}{Z} x^{s} y^{t}\left|\mathscr{P}_{m, n, s, t}\right| \\
& =\frac{1}{Z} \sum_{N \geq 0} \sum_{\substack{\lambda \in N \\
\lambda_{1} \leq h}} Q_{\lambda}(x) P_{\lambda}(y) .
\end{aligned}
$$

(Here $\mathrm{P}_{m, n}$ denotes probability before $\alpha$-specialization.) Thus, by (4.3),

$$
\mathrm{P}_{m, n}(L \leq h)=\frac{1}{Z}\left(\operatorname{det}\left(I-K_{h}(x) K_{h}(y)\right)\right)^{1 / 2} .
$$

The above uses the combinatorial definition (2.1) of the Schur $Q$-function. The reason for the occurence of $P_{\lambda}(y)$ (instead of $Q_{\lambda}(y)$ ) is that the recording tableau $T$ has no 
marked elements on the diagonal, which accounts for the factor $2^{-\ell(\lambda)}$. (There are exactly $2^{\ell(\lambda)}$ entries on the main diagonal in a marked shifted tableau of shape $\lambda$, and so there are $2^{\ell(\lambda)}$ ways to mark and unmark the diagonal elements.)

Observe the consequence that the distribution function $\mathrm{P}_{m, n}(L \leq h)$ is a symmetric function of $x=\left(x_{1}, \ldots, x_{m}\right)$ and of $y=\left(y_{1}, \ldots, y_{n}\right)$.

\section{Proof of the main theorem}

\subsection{An operator formulation}

We begin by deriving an alternative representation for $\operatorname{det}\left(I-K_{h}(x) K_{h}(y)\right)$ in terms of Toeplitz and Hankel operators on the Hilbert space $\ell^{2}\left(\mathbb{Z}_{+}\right)\left(\mathbb{Z}_{+}:=\mathbb{N} \cup 0\right)$. These may well be of independent interest in the theory of Schur $Q$-functions. We assume at first only that $x, y \in \ell^{1}$ together with uniform estimates $x_{j}, y_{j} \leq c<1$

To set notation, we let $\left\{e_{j}\right\}_{j \geq 0}$ denote the canonical basis of $\ell^{2}\left(\mathbb{Z}_{+}\right)$. Since the vector $e_{0}$ occurs frequently, we denote $e_{0}$ by $e$, and it is convenient to set $e_{-1}=0$. The backward shift operator $\Lambda$ is characterized by

$$
\Lambda e_{j}=e_{j-1},
$$

and its adjoint $\Lambda^{*}$ is the forward shift operator. The two satisfy

$$
\Lambda \Lambda^{*}=I \quad \text { and } \quad \Lambda^{*} \Lambda=I-e \otimes e,
$$

where for vectors $u$ and $v$ we denote by $u \otimes v$ the operator sending a vector $f$ to $u(v, f)$.

Suppressing temporarily the parameters $x$ and $y$, we define $L$ to be the matrix with entries

$$
L_{j k}=\left(\frac{1}{2 \pi \mathrm{i}}\right)^{2} \iint \frac{Q(z) Q(\zeta)}{z^{j+1} \zeta^{k+1}} \frac{\mathrm{d} z \mathrm{~d} \zeta}{z+\zeta},
$$

where $Q$ is defined as in (2.4). Here the contours can be taken to be concentric circles of different radii near the unit circle. Since $Q(z) Q(-z)=1$, the residue at $\zeta=-z$ in the integral defining $L$ is zero, so we may freely choose whether the $z$-contour lies inside or outside the $\zeta$-contour without affecting the value of the integral. (Equivalently, $L$ is symmetric.)

From (2.5), we see that

$$
Q_{(j, k)}=L_{j-1, k}-L_{j, k-1},
$$

where we set $L_{-1, k}=0$. Note that $L_{j-1,0}=q_{j}$. The matrix elements $K_{j k}$ given in 
(4.2) are then

$$
K_{j k}= \begin{cases}-\frac{1}{\sqrt{2}} L_{0, k-1}, & j=0, k \neq 0, \\ \frac{1}{\sqrt{2}} L_{j-1,0}, & j \neq 0, k=0, \\ \frac{1}{2}\left(L_{j-1, k}-L_{j, k-1}\right), & j, k>0 .\end{cases}
$$

By introducing the vector $q=q(x)=\left(q_{0}(x), q_{1}(x), \ldots\right)$, the operator $K$ can be written

$$
K=\frac{1}{2}\left(\Lambda^{*} L-L \Lambda\right)+\frac{1}{2} \omega(e \otimes q-q \otimes e),
$$

where $\omega=1-\sqrt{2}$.

The operator $L$ is expressible in terms of Toeplitz and Hankel matrices acting on $\ell^{2}\left(\mathbb{Z}_{+}\right)$. Recall that $T(\psi)$, the Toeplitz matrix with symbol $\psi$, has $(j, k)$-entry $\psi_{j-k}$ (subscripts denote Fourier coefficients here), while the Hankel matrix $H(\psi)$ has $(j, k)$-entry $\psi_{j+k+1}$. If we assume that the contours in (6.2) are chosen so that $|\zeta|<|z|$ and expand $(z+\zeta)^{-1}$ in powers of $\zeta / z$, we obtain

$$
L_{i j}=\sum_{k=0}^{\infty}(-1)^{k}\left(\frac{1}{2 \pi \mathrm{i}}\right)^{2} \iint z^{-i-k-2} \zeta^{k-j-1} Q(z) Q(\zeta) \mathrm{d} z \mathrm{~d} \zeta .
$$

Now make the substitution $\zeta \rightarrow-\zeta^{-1}$ to obtain

$$
(-1)^{j} \sum_{k=0}^{\infty}\left(\frac{1}{2 \pi \mathrm{i}}\right)^{2} \iint z^{-i-k-2} \zeta^{-k+j-1} \frac{Q(z)}{\widetilde{Q}(\zeta)} \mathrm{d} z \mathrm{~d} \zeta,
$$

where $\widetilde{Q}(\zeta)=Q\left(\zeta^{-1}\right)$. The $z$-integral gives $Q_{i+k+1}$, while the $\zeta$-integral gives $\left(\widetilde{Q}^{-1}\right)_{k-j}$. It follows that

$$
L=H(Q) T\left(\widetilde{Q}^{-1}\right) J
$$

where $J$ is the diagonal matrix with diagonal entries $(-1)^{j}$. If in the last integrals we make the substitutions $z \rightarrow-z, \zeta \rightarrow-\zeta$, we find that also

$$
L=-J H\left(Q^{-1}\right) T(\widetilde{Q}) .
$$

If we reintroduce our parameters $x$ and $y$, which we now write for notational convenience as subscripts, and use the two representations of $L$, we see that

$$
\begin{aligned}
\operatorname{det}\left(I+L_{x} L_{y}\right) & =\operatorname{det}\left(I-H\left(Q_{x}\right) T\left(\widetilde{Q}_{x}^{-1}\right) H\left(Q_{y}^{-1}\right) T\left(\widetilde{Q}_{y}\right)\right) \\
& =\operatorname{det}\left(I-T\left(\widetilde{Q}_{y}\right) H\left(Q_{x}\right) T\left(\widetilde{Q}_{x}^{-1}\right) H\left(Q_{y}^{-1}\right)\right) .
\end{aligned}
$$

Here we have used the general identity $\operatorname{det}(I-A B)=\operatorname{det}(I-B A)$, valid if one of the operators is trace class and the other bounded, and the fact that the Hankel 
operators are Hilbert-Schmidt under our assumptions on $x$ and $y$. Another general fact is

$$
T\left(\psi_{1}\right) H\left(\psi_{2}\right)+H\left(\psi_{1}\right) T\left(\tilde{\psi}_{2}\right)=H\left(\psi_{1} \psi_{2}\right) .
$$

In particular, if $\psi_{1}$ is a minus function (Fourier coefficients with positive index all vanish), then $T\left(\psi_{1}\right) H\left(\psi_{2}\right)=H\left(\psi_{1} \psi_{2}\right)$. From this we find that

$$
T\left(\widetilde{Q}_{y}\right) H\left(Q_{x}\right)=H\left(Q_{x} \widetilde{Q}_{y}\right) \quad \text { and } \quad T\left(\widetilde{Q}_{x}^{-1}\right) H\left(Q_{y}^{-1}\right)=H\left(\widetilde{Q}_{x}^{-1} Q_{y}^{-1}\right),
$$

so the product of these equals $H(\phi) H\left(\widetilde{\phi}^{-1}\right)$, where

$$
\phi(z):=Q_{x}(z) \widetilde{Q}_{y}(z) .
$$

Since yet another general identity is

$$
T\left(\psi_{1}\right) T\left(\psi_{2}\right)=T\left(\psi_{1} \psi_{2}\right)-H\left(\psi_{1}\right) H\left(\tilde{\psi}_{2}\right),
$$

we have $I-H(\phi) H\left(\widetilde{\phi}^{-1}\right)=T(\phi) T\left(\phi^{-1}\right)$, and we have shown that

$$
\operatorname{det}\left(I+L_{x} L_{y}\right)=\operatorname{det} T(\phi) T\left(\phi^{-1}\right) .
$$

If a symbol $\phi$ has geometric mean 1 and is sufficiently well behaved, then the strong Szegö limit theorem says that

$$
\lim _{h \rightarrow \infty} \operatorname{det} T_{h}(\phi)=E(\phi):=\exp \left(\sum_{n=1}^{\infty} n(\log \phi)_{n}(\log \phi)_{-n}\right),
$$

where $T_{h}(\phi)=\left(\phi_{j-k}\right)_{j, k=0, \ldots, h-1}$. In the case of our symbol given by (6.7), we find that

$$
E(\phi)=\left(\prod_{i, j} \frac{1+x_{i} y_{j}}{1-x_{i} y_{j}}\right)^{2}=Z^{2},
$$

where $Z$ is as in the right side of (2.3). But there is another formula for $E(\phi)$, namely (see $[\mathrm{W}])$,

$$
E(\phi)=\operatorname{det} T(\phi) T\left(\phi^{-1}\right),
$$

and so from (6.9) we have the identity

$$
\operatorname{det}\left(I+L_{x} L_{y}\right)=Z^{2} .
$$

In the case of Schur functions, the right-hand side of the Gessel identity is a Toeplitz determinant, and the Cauchy identity for Schur functions emerges as a consequence of the Szegö limit theorem. In view of the last identity and the connection between the operators $L$ and $K$ on $\ell^{2}\left(\mathbb{Z}_{+}\right)$, it is tempting to try to find, using these, an independent derivation of (2.3). It follows from (6.3) that $I-K(x) K(y)$ and 
$I+L_{x} L_{y}$ differ by a finite-rank operator. This operator cannot contribute to the determinant, but we do not see, a priori, why this is so. So such an independent derivation eludes us.

To continue now, we let $P_{h}$ be the projection operator onto the subspace of $\ell^{2}\left(\mathbb{Z}_{+}\right)$ spanned by $\left\{e_{0}, e_{1}, \ldots, e_{h}\right\}$. Thus if $K$ is the operator on $\ell^{2}\left(\mathbb{Z}_{+}\right)$, then $K_{h}=P_{h} K P_{h}$. Instead of working directly with the product $K(x) K(y)$, it is convenient to write $(2 \times 2)$-matrices with operator entries. Thus $\operatorname{det}\left(I-K_{h}(x) K_{h}(y)\right)$ is the determinant of

$$
\left(\begin{array}{cc}
P_{h} & 0 \\
0 & P_{h}
\end{array}\right)\left(\begin{array}{cc}
I & K(x) \\
K(y) & I
\end{array}\right)\left(\begin{array}{cc}
P_{h} & 0 \\
0 & P_{h}
\end{array}\right)
$$

thought of as acting on $P_{h} \ell^{2}\left(\mathbb{Z}_{+}\right) \oplus P_{h} \ell^{2}\left(\mathbb{Z}_{+}\right)$. To simplify notation, we use $P_{h}$ to denote also

$$
\left(\begin{array}{cc}
P_{h} & 0 \\
0 & P_{h}
\end{array}\right)
$$

and we set

$$
\mathscr{K}=\left(\begin{array}{cc}
0 & K(x) \\
K(y) & 0
\end{array}\right)
$$

Thus

$$
\operatorname{det}\left(I-K_{h}(x) K_{h}(y)\right)=\operatorname{det} P_{h}(I+\mathscr{K}) P_{h} .
$$

It follows from (4.4) and the infinite-dimensional version of Jacobi's theorem on the principal $(n \times n)$-minor of the inverse of a (finite) matrix* that this may be written

$$
\operatorname{det}\left(I-K_{h}(x) K_{h}(y)\right)=Z^{2} \operatorname{det}\left(\left(I-P_{h}\right)(I+\mathscr{K})^{-1}\left(I-P_{h}\right)\right) .
$$

Thus our first goal is to compute $(I+\mathscr{K})^{-1}$.

Using the easily verified fact

$$
\Lambda^{*} L+L \Lambda=q \otimes q-e \otimes e
$$

and (6.3), we see that $K=\Lambda^{*} L+R^{-}=-L \Lambda+R^{+}$, where

$$
R^{ \pm}=\frac{1}{2}( \pm q \otimes q \mp e \otimes e+\omega(e \otimes q-q \otimes e)) .
$$

Thus

$$
I+\mathscr{K}=\left(\begin{array}{cc}
I & \Lambda^{*} L_{x}+R_{x}^{-} \\
\Lambda^{*} L_{y}+R_{y}^{-} & I,
\end{array}\right),
$$

where subscripts have the usual meaning.

*The infinite-dimensional result follows by replacing the operator $\mathscr{K}$ by $P_{N} \mathscr{K} P_{N}$, applying the finitedimensional result, and taking the $(N \rightarrow \infty)$-limit. We use the fact that $\mathscr{K}$ is trace class, which holds since the Hankel operators are trace class. Of course, all this requires that the infinite-dimensional operator be invertible. This follows from the limit results we establish, as we see at the end of $\S 6.3$. 


\subsection{Calculation of $(I+\mathscr{K})^{-1}$}

The fundamental objects that appear here are $I+L_{x} L_{y}$ and $I+L_{y} L_{x}$, and we begin by showing that they are invertible and computing their inverses. Define

$$
H_{1}=H\left(Q_{x} \widetilde{Q}_{y}^{-1}\right) \quad \text { and } \quad H_{2}=H\left(Q_{y} \widetilde{Q}_{x}^{-1}\right) .
$$

We prove the basic identities

$$
\left(I+L_{x} L_{y}\right)^{-1}=I-H_{1} H_{2} \quad \text { and } \quad\left(I+L_{y} L_{x}\right)^{-1}=I-H_{2} H_{1} .
$$

We do this with the help of (6.6) and (6.8). Using these and (6.4), we find that

$$
\begin{aligned}
H_{1} H_{2} L_{x} & =H\left(Q_{x} \widetilde{Q}_{y}^{-1}\right) H\left(Q_{y} \widetilde{Q}_{x}^{-1}\right) H\left(Q_{x}\right) T\left(\widetilde{Q}_{x}^{-1}\right) J \\
& =H\left(Q_{x} \widetilde{Q}_{y}^{-1}\right)\left[T\left(Q_{y}\right)-T\left(Q_{y} \widetilde{Q}_{x}^{-1}\right) T\left(\widetilde{Q}_{x}\right)\right] T\left(\widetilde{Q}_{x}^{-1}\right) J \\
& =\left[H\left(Q_{x}\right) T\left(\widetilde{Q}_{x}^{-1}\right)-H\left(Q_{x} \widetilde{Q}_{y}^{-1}\right) T\left(Q_{y} \widetilde{Q}_{x}^{-1}\right)\right] J \\
& =L_{x}-H_{1} T\left(Q_{y} \widetilde{Q}_{x}^{-1}\right) J .
\end{aligned}
$$

Thus

$$
\left(I-H_{1} H_{2}\right) L_{x}=H_{1} T\left(Q_{y} \widetilde{Q}_{x}^{-1}\right) J .
$$

From (6.14) and (6.5) we obtain similarly

$$
\begin{aligned}
\left(I-H_{1} H_{2}\right) L_{x} L_{y} & =-H_{1} T\left(Q_{y} \widetilde{Q}_{x}^{-1}\right) H\left(Q_{y}^{-1}\right) T\left(\widetilde{Q}_{y}\right) \\
& =H_{1} H\left(Q_{y} \widetilde{Q}_{x}^{-1}\right) T\left(\widetilde{Q}_{y}^{-1}\right) T\left(\widetilde{Q}_{y}\right)=H_{1} H_{2} .
\end{aligned}
$$

This establishes the first identity of (6.13), and the second is obtained by interchanging $x$ and $y$.

Beginning the calculation of $(I+\mathscr{K})^{-1}$, we refer to (6.11) and (6.12) and find that

$$
\left(\begin{array}{cc}
I & L_{x} \Lambda \\
L_{y} \Lambda & I
\end{array}\right)(I+\mathscr{K})=\left(\begin{array}{cc}
I+L_{x} L_{y} & 0 \\
0 & I+L_{y} L_{x}
\end{array}\right)+\left(\begin{array}{cc}
L_{x} \Lambda R_{y}^{-} & R_{x}^{+} \\
R_{y}^{+} & L_{y} \Lambda R_{x}^{-},
\end{array}\right),
$$

where we use subscripts as before. Using (6.13), this may be written

$$
\left(\begin{array}{cc}
I+L_{x} L_{y} & 0 \\
0 & I+L_{y} L_{x}
\end{array}\right)\left[I+\left(\begin{array}{cc}
\left(I-H_{1} H_{2}\right) L_{x} \Lambda R_{y}^{-} & \left(I-H_{1} H_{2}\right) R_{x}^{+} \\
\left(I-H_{2} H_{1}\right) R_{y}^{+} & \left(I-H_{2} H_{1}\right) L_{y} \Lambda R_{x}^{-}
\end{array}\right)\right] .
$$


Hence

$$
\begin{aligned}
(I+\mathscr{K})^{-1}= & {\left[I+\left(\begin{array}{cc}
\left(I-H_{1} H_{2}\right) L_{x} \Lambda R_{y}^{-} & \left(I-H_{1} H_{2}\right) R_{x}^{+} \\
\left(I-H_{2} H_{1}\right) R_{y}^{+} & \left(I-H_{2} H_{1}\right) L_{y} \Lambda R_{x}^{-}
\end{array}\right)\right]^{-1} } \\
& \times\left(\begin{array}{cc}
I-H_{1} H_{2} & \left(I-H_{1} H_{2}\right) L_{x} \Lambda \\
\left(I-H_{2} H_{1}\right) L_{y} \Lambda & I-H_{2} H_{1}
\end{array}\right) .
\end{aligned}
$$

To compute the entries of the matrix inside the large bracket, we show that

$$
\left(I-H_{1} H_{2}\right) L_{x} \Lambda q_{y}=H_{1} H_{2} e \quad \text { and } \quad\left(I-H_{1} H_{2}\right) q_{x}=T_{1} e,
$$

where we set

$$
T_{1}=T\left(Q_{x} \widetilde{Q}_{y}^{-1}\right) \quad \text { and } \quad T_{2}=T\left(Q_{y} \widetilde{Q}_{x}^{-1}\right) .
$$

For the first we use the fact that $\Lambda q=L e$, which gives

$$
\left(I-H_{1} H_{2}\right) L_{x} \Lambda q_{y}=\left(I-H_{1} H_{2}\right) L_{x} L_{y} e=H_{1} H_{2} e .
$$

To derive the second we use the fact that $q_{x}=T\left(Q_{x}\right) e$ and compute

$$
\begin{aligned}
H_{1} H_{2} T\left(Q_{x}\right) & =H\left(Q_{x} \widetilde{Q}_{y}^{-1}\right) H\left(Q_{y} \widetilde{Q}_{x}^{-1}\right) T\left(Q_{x}\right)=H\left(Q_{x} \widetilde{Q}_{y}^{-1}\right) H\left(Q_{y}\right) \\
& =T\left(Q_{x}\right)-T\left(Q_{x} \widetilde{Q}_{y}^{-1}\right) T\left(\widetilde{Q}_{y}\right) .
\end{aligned}
$$

Since $T\left(\widetilde{Q}_{y}\right) e=e$ (the matrix is upper-triangular with $(0,0)$-entry 1$)$, this gives

$$
H_{1} H_{2} q_{x}=q_{x}-T_{1} e,
$$

which is equivalent to the desired identity. Of course, the same identities hold if we make the interchanges $x \leftrightarrow y$ and $1 \leftrightarrow 2$.

With these identities and the fact that $\Lambda R^{-}=-(1 / 2)-\Lambda q \otimes(q+\omega e)$, we find that the matrix in large brackets may be written

$$
I+\frac{1}{2}\left(\begin{array}{cc}
-H_{1} H_{2} e \otimes\left(q_{y}+\omega e\right) & T_{1} e \otimes\left(q_{x}-\omega e\right)+\left(I-H_{1} H_{2}\right) e \otimes\left(\omega q_{x}-e\right) \\
T_{2} e \otimes\left(q_{y}-\omega e\right)+\left(I-H_{2} H_{1}\right) e \otimes\left(\omega q_{y}-e\right) & -H_{2} H_{1} e \otimes\left(q_{x}+\omega e\right)
\end{array}\right) .
$$

This in turn has the form

$$
I+\sum_{i=1}^{4} a_{i} \otimes b_{i}
$$


where

$$
\begin{array}{cc}
a_{1}=\frac{1}{2}\left(\begin{array}{c}
-H_{1} H_{2} e \\
-\omega H_{2} H_{1} e+T_{2} e+\omega e
\end{array}\right), & a_{2}=\frac{1}{2}\left(\begin{array}{c}
-\omega H_{1} H_{2} e+T_{1} e+\omega e \\
-H_{2} H_{1} e
\end{array}\right), \\
a_{3}=\frac{1}{2}\left(\begin{array}{c}
-\omega H_{1} H_{2} e \\
H_{2} H_{1} e-\omega T_{2} e-e
\end{array}\right), & a_{4}=\frac{1}{2}\left(\begin{array}{c}
H_{1} H_{2} e-\omega T_{1} e-e \\
-\omega H_{2} H_{1} e
\end{array}\right), \\
b_{1}=\left(\begin{array}{c}
q_{y} \\
0
\end{array}\right), \quad b_{2}=\left(\begin{array}{c}
0 \\
q_{x}
\end{array}\right), & b_{3}=\left(\begin{array}{l}
e \\
0
\end{array}\right), \quad b_{4}=\left(\begin{array}{l}
0 \\
e
\end{array}\right) .
\end{array}
$$

At this stage we have shown that

$$
(I+\mathscr{K})^{-1}=\left(I+\sum_{i=1}^{4} a_{i} \otimes b_{i}\right)^{-1}\left(\begin{array}{cc}
I-H_{1} H_{2} & \left(I-H_{1} H_{2}\right) L_{x} \Lambda \\
\left(I-H_{2} H_{1}\right) L_{y} \Lambda & I-H_{2} H_{1}
\end{array}\right) .
$$

If we have a finite-rank operator $\sum a_{i} \otimes b_{i}$, then

$$
\left(I+\sum a_{i} \otimes b_{i}\right)^{-1}=I-\sum_{i, j}\left(S^{-1}\right)_{i j} a_{i} \otimes b_{j},
$$

where $S$ is the matrix with entries

$$
S_{i j}=\delta_{i j}+\left(b_{i}, a_{j}\right)
$$

In our case we have to compute 16 inner products, which is not as bad as it might seem since there are basic inner products from which the others can be derived. And if we have evaluated any inner product, then we have evaluated another with the interchanges $x \leftrightarrow y$ and $1 \leftrightarrow 2$. Two basic inner products are trivial:

$$
(e, e)=1 \quad \text { and } \quad\left(e, q_{y}\right)=1 .
$$

Two are not evaluable in simpler terms but just notationally. We set

$$
t=\left(T_{1} e, e\right)=\left(T_{2} e, e\right) \quad \text { and } \quad h=\left(H_{1} H_{2} e, e\right)=\left(H_{2} H_{1} e, e\right) .
$$

(The equality of the first two inner products follows from the fact that $T_{1}^{*}=J T_{2} J$ and $J e=e$.) The nontrivial ones are

$$
\left(T_{1} e, q_{y}\right)=1 \quad \text { and } \quad\left(H_{1} H_{2} e, q_{y}\right)=1-t .
$$


For the first, we have $\left(T_{1} e, q_{y}\right)=\left(T_{1} e, T\left(Q_{y}\right) e\right)=\left(T\left(\widetilde{Q}_{y}\right) T_{1} e, e\right)$, and this is the $(0,0)$-entry of $T\left(\widetilde{Q}_{y}\right) T\left(Q_{x} \widetilde{Q}_{y}^{-1}\right)=T\left(Q_{x}\right)$. The $(0,0)$-entry equals 1 . For the second, we have

$$
\left(H_{1} H_{2} e, q_{y}\right)=1-\left(\left(I-H_{1} H_{2}\right) e, q_{y}\right)=1-\left(e,\left(I-H_{2} H_{1}\right) q_{y}\right)=1-\left(e, T_{2} e\right)
$$

by the second identity of (6.15).

We can now write down all 16 inner products. For convenience, we multiply them by 2 :

$$
\begin{gathered}
2\left(a_{1}, b_{1}\right)=-1+t, \quad 2\left(a_{2}, b_{1}\right)=\omega t+1, \\
2\left(a_{3}, b_{1}\right)=-\omega(1-t), \quad 2\left(a_{4}, b_{1}\right)=-t-\omega-2, \\
2\left(a_{1}, b_{2}\right)=\omega t+1, \quad 2\left(a_{2}, b_{2}\right)=-1+t, \\
2\left(a_{3}, b_{2}\right)=-t-\omega-2, \quad 2\left(a_{4}, b_{2}\right)=-\omega(1-t), \\
2\left(a_{1}, b_{3}\right)=-h, \quad 2\left(a_{2}, b_{3}\right)=-\omega h+t+\omega, \\
2\left(a_{3}, b_{3}\right)=-\omega h, \quad 2\left(a_{4}, b_{3}\right)=h-\omega t-1, \\
2\left(a_{1}, b_{4}\right)=-\omega h+t+\omega, \quad 2\left(a_{2}, b_{4}\right)=-h, \\
2\left(a_{3}, b_{4}\right)=h-\omega t-1, \quad 2\left(a_{4}, b_{4}\right)=-\omega h .
\end{gathered}
$$

Let us see which vectors arise in the end. From (6.16) and (6.17),

$$
(I+\mathscr{K})^{-1}=\left(\begin{array}{cc}
I-H_{1} H_{2} & \left(I-H_{1} H_{2}\right) L_{x} \Lambda \\
\left(I-H_{2} H_{1}\right) L_{y} \Lambda & I-H_{2} H_{1}
\end{array}\right)-\sum_{i, j=1}^{4} s_{i j} a_{i} \otimes b_{j}^{\prime},
$$

where $s_{i j}=\left(S^{-1}\right)_{i j}$ and

$$
b_{j}^{\prime}=\left(\begin{array}{cc}
I-H_{2} H_{1} & \Lambda^{*} L_{y}\left(I-H_{1} H_{2}\right) \\
\Lambda^{*} L_{x}\left(I-H_{2} H_{1}\right) & I-H_{1} H_{2}
\end{array}\right) b_{j} .
$$

The quantities that appear in the $a_{i}$, other than $e$, which we can ignore since ( $I-$ $\left.P_{h}\right) e=0$, are $H_{1} H_{2} e$ and $T_{1} e$ in the first component and $H_{2} H_{1} e$ and $T_{2} e$ in the second. Those in the $b_{j}$ are $q_{y}$ and $e$ in the first component and $q_{x}$ and $e$ in the second. For $b_{j}^{\prime}$ we use (6.15) to see that $T_{2} e, H_{2} H_{1} e, \Lambda^{*} L_{y} T_{1} e$, and $\Lambda^{*} L_{y}\left(I-H_{1} H_{2}\right) e$ appear in the first component and $T_{1} e, H_{1} H_{2} e, \Lambda^{*} L_{x} T_{2} e$, and $\Lambda^{*} L_{x}\left(I-H_{2} H_{1}\right) e$ in the second. (The $e$ that appear once again drop out in the end.)

Two new vectors appear here (as well as those obtained by the usual interchanges). We claim that $\Lambda^{*} L_{y} T_{1} e=T_{2} e-t e \quad$ and $\quad \Lambda^{*} L_{y}\left(I-H_{1} H_{2}\right) e=H_{2} H_{1} e+t T_{2} e-(1-h) e$. 
For the first, we have

$$
\begin{aligned}
L_{y} T_{1} & =-J H\left(Q_{y}^{-1}\right) T\left(\widetilde{Q}_{y}\right) T\left(Q_{x} \widetilde{Q}_{y}^{-1}\right)=-J H\left(Q_{y}^{-1}\right) T\left(Q_{x}\right) \\
& =-J H\left(\widetilde{Q}_{x} Q_{y}^{-1}\right)=H\left(Q_{y} \widetilde{Q}_{x}^{-1}\right) J,
\end{aligned}
$$

so

$$
\Lambda^{*} L_{y} T_{1} e=\Lambda^{*} H\left(Q_{y} \widetilde{Q}_{x}^{-1}\right) e=T_{2} e-t e .
$$

For the second, we take transposes and interchange $x$ and $y$ in (6.14) to obtain

$$
\begin{aligned}
L_{y} T_{1} & =-J H\left(Q_{y}^{-1} L_{y}\left(1-H_{1} H_{2}\right) e=J T\left(\widetilde{Q}_{x} Q_{y}^{-1}\right) H\left(Q_{y} \widetilde{Q}_{x}^{-1}\right) e\right. \\
& =-T\left(Q_{y} \widetilde{Q}_{x}^{-1}\right) H\left(\widetilde{Q}_{x} Q_{y}^{-1}\right) e=H\left(Q_{y} \widetilde{Q}_{x}^{-1}\right) T\left(Q_{x} \widetilde{Q}_{y}^{-1}\right) e .
\end{aligned}
$$

So

$$
\begin{aligned}
\Lambda^{*} L_{y}\left(1-H_{1} H_{2}\right) e & =\left[H\left(z Q_{y} \widetilde{Q}_{x}^{-1}\right)-e \otimes T_{2} e\right] T\left(Q_{x} \widetilde{Q}_{y}^{-1}\right) e \\
& =H\left(z Q_{y} \widetilde{Q}_{x}^{-1}\right) H\left(z Q_{x} \widetilde{Q}_{y}^{-1}\right) e-\left(T_{1} e, T_{2} e\right) e \\
& =H_{2} H_{1} e+\left(T_{2} e \otimes T_{1} e\right) e-\left(T_{1} e, T_{2} e\right) e .
\end{aligned}
$$

The next-to-last term equals $t T_{2} e$, while the last inner product equals

$$
\left(J T_{2}^{*} J J T_{1} J e, e\right)=\left(T\left(Q_{x} \widetilde{Q}_{y}^{-1}\right) T\left(\widetilde{Q}_{y} Q_{x}^{-1}\right) e, e\right)=\left(\left(I-H_{1} H_{2}\right) e, e\right) .
$$

This establishes the second claim.

It follows from the above that the only vectors that arise in the $b_{j}^{\prime}$ are $T_{2} e$ and $\mathrm{H}_{2} \mathrm{H}_{1} e$ in the first component and $T_{1} e$ and $H_{1} H_{2} e$ in the second.

\subsection{Specialization}

At this point we impose the $\alpha$-specialization. Thus the first $m x_{i}$ and the first $n y_{i}$ are equal to $\alpha$ and the rest are equal to zero. We assume that $\tau=m / n$ is a constant satisfying $\alpha^{2}<\tau<\alpha^{-2}$, and we first determine the asymptotics as $n \rightarrow \infty$ of the quantities appearing in the inner products. We claim that

$$
\lim _{n \rightarrow \infty} t=0 \quad \text { and } \quad \lim _{n \rightarrow \infty} h=\frac{1}{2} .
$$

For the first, we have

$$
t=\left(T_{1}\right)_{0,0}=\frac{1}{2 \pi \mathrm{i}} \int\left(\frac{1+\alpha z}{1-\alpha z}\right)^{m}\left(\frac{z-\alpha}{z+\alpha}\right)^{n} \frac{\mathrm{d} z}{z} .
$$

If we apply steepest descent, we see that the saddle points should satisfy

$$
\frac{\tau}{1-\alpha^{2} z^{2}}+\frac{1}{z^{2}-\alpha^{2}}=0
$$




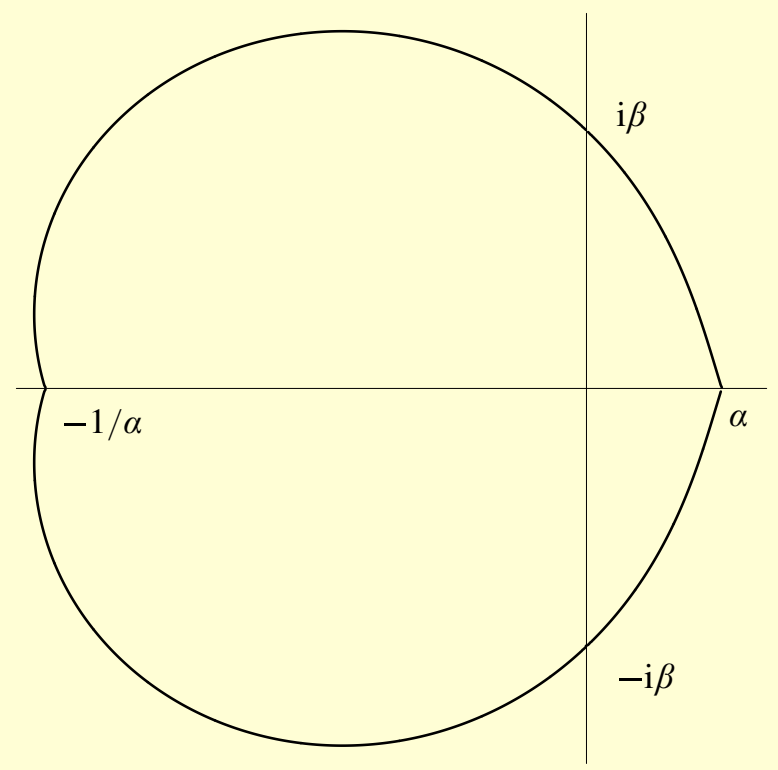

Figure 1. Steepest descent curve

and so are given by

$$
z= \pm \mathrm{i} \sqrt{\frac{1-\alpha^{2} \tau}{\tau-\alpha^{2}}}= \pm \mathrm{i} \beta .
$$

These are purely imaginary under our assumption on $\tau$. The steepest descent curve passes through these points and closes at $\alpha$ and $-\alpha^{-1}$ (see Fig. 1). The integral is $O\left(n^{-1 / 2}\right)$. For the second, we have

$$
\begin{aligned}
\left(H_{1} H_{2}\right)_{i, j}= & \left(\frac{1}{2 \pi \mathrm{i}}\right)^{2} \sum_{k=0}^{\infty} \iint\left(\frac{1+\alpha z}{1-\alpha z}\right)^{m}\left(\frac{z-\alpha}{z+\alpha}\right)^{n}\left(\frac{1+\alpha \zeta}{1-\alpha \zeta}\right)^{n}\left(\frac{\zeta-\alpha}{\zeta+\alpha}\right)^{m} \\
& \times z^{-i-k-2} \zeta^{-k-j-2} \mathrm{~d} z \mathrm{~d} \zeta \\
= & \left(\frac{1}{2 \pi \mathrm{i}}\right)^{2} \iint\left(\frac{1+\alpha z}{1-\alpha z}\right)^{m}\left(\frac{z-\alpha}{z+\alpha}\right)^{n}\left(\frac{1+\alpha \zeta}{1-\alpha \zeta}\right)^{n}\left(\frac{\zeta-\alpha}{\zeta+\alpha}\right)^{m} z^{-i-1} \\
& \times \zeta^{-j-1} \frac{\mathrm{d} z \mathrm{~d} \zeta}{z \zeta-1},
\end{aligned}
$$

where the contours are such that $|z \zeta|>1$. Setting $i=j=0$ and making the substitution $\zeta \rightarrow \zeta^{-1}$ gives

$$
\begin{aligned}
h & =\left(H_{1} H_{2}\right)_{0,0} \\
& =\left(\frac{1}{2 \pi \mathrm{i}}\right)^{2} \iint\left(\frac{1+\alpha z}{1-\alpha z}\right)^{m}\left(\frac{z-\alpha}{z+\alpha}\right)^{n}\left(\frac{\zeta+\alpha}{\zeta-\alpha}\right)^{n}\left(\frac{1-\alpha \zeta}{1+\alpha \zeta}\right)^{m} \frac{\mathrm{d} z \mathrm{~d} \zeta}{z(z-\zeta)},
\end{aligned}
$$


where now on the contours (both still described counterclockwise) $|z|>|\zeta|$. If we ignore the $z-\zeta$ in the denominator, we have two integrals, to each of which we apply steepest descent. The saddle points are $\pm \mathrm{i} \beta$ for both. The new $z$-contour is as before, but the new $\zeta$-contour closes at $-\alpha$ and $\alpha^{-1}$. We first deform the original $\zeta$-contour to this, always remaining inside the original $z$-contour. Then we deform the $z$-contour to its steepest descent curve. In the process we pass through the points of the $\zeta$-contour from $-\mathrm{i} \beta$ to $\mathrm{i} \beta$ in the right half-plane. The $z$ residues at these points are $1 / \zeta$, and so the deformations lead to the double integral over the steepest descent contours, which is $O\left(n^{-1 / 2}\right)$, plus $(2 \pi i)^{-1} \int_{-\mathrm{i} \beta}^{\mathrm{i} \beta} \mathrm{d} \zeta / \zeta=1 / 2$. This establishes the second limit.

When $\omega=1-\sqrt{2}$, we find that det $S$ has the limit $(5-3 \sqrt{2}) / 8 \neq 0$ as $t \rightarrow 0$, $h \rightarrow 1 / 2$. $^{*}$ Hence the entries of $S^{-1}$ are all bounded. (The invertibility of $S$ for large $n$ implies in turn the invertibility of $I+\mathscr{K}$ and hence the validity of the determinant identities we have been using.)

\subsection{Scaling}

If we write $P$ for $I-P_{h}$, then we see from (6.18) that $P(I+\mathscr{K})^{-1} P$ equals the identity operator $I$ minus

$$
\begin{aligned}
\left(\begin{array}{cc}
P H_{1} H_{2} P & 0 \\
0 & P H_{2} H_{1} P
\end{array}\right) & -\left(\begin{array}{cc}
0 & P\left(I-H_{1} H_{2}\right) L_{x} \Lambda P \\
P\left(I-H_{2} H_{1}\right) L_{y} \Lambda P & 0
\end{array}\right) \\
& +\sum_{i, j=1}^{4} s_{i j} P a_{i} \otimes P b_{j}^{\prime} .
\end{aligned}
$$

Eventually we set $i=h+n^{1 / 3} x, j=h+n^{1 / 3} y$, where $h=c n+n^{1 / 3} s$ with $c$ to be determined. The operators $P H_{1}$ and $H_{1} P$ give rise to integrals like

$$
\int\left(\frac{1+\alpha z}{1-\alpha z}\right)^{m}\left(\frac{z-\alpha}{z+\alpha}\right)^{n} z^{-c n-n^{1 / 3} x} \mathrm{~d} z
$$

(with a different $x$ ), and $P H_{2}$ and $H_{2} P$ give rise to integrals like

$$
\int\left(\frac{1+\alpha z}{1-\alpha z}\right)^{n}\left(\frac{z-\alpha}{z+\alpha}\right)^{m} z^{-c n-n^{1 / 3} x} \mathrm{~d} z .
$$

If we make the substitution $z \rightarrow z^{-1}$ in the latter, we get an integral like

$$
\int\left(\frac{z+\alpha}{z-\alpha}\right)^{n}\left(\frac{1-\alpha z}{1+\alpha z}\right)^{m} z^{c n+n^{1 / 3} x} \mathrm{~d} z
$$

*Computations verify that when $\tau<\alpha^{2}$, the limit of $h$ is zero and $t=(-1)^{m}+o(1)$, and that the limit of det $S$ is $(9-4 \sqrt{2}) / 2$ as $m \rightarrow \infty$ through even values and $-1 / 2$ as $m \rightarrow \infty$ through odd values. For the limits when $\tau>\alpha^{-2}$, we replace $m$ by $n$. The proofs should be similar to what we have already done, except that the saddle points will now be real. 
If we set

$$
\psi(z)=\left(\frac{1+\alpha z}{1-\alpha z}\right)^{m}\left(\frac{z-\alpha}{z+\alpha}\right)^{n} z^{-c n}
$$

our integrals become

$$
\int \psi(z) z^{-n^{1 / 3} x} \mathrm{~d} z \quad \text { and } \quad \int \psi(z)^{-1} z^{n^{1 / 3} x} \mathrm{~d} z .
$$

If we think of the factors $\psi(z)^{ \pm 1}$ as the dominant ones and apply steepest descent, there are in general two saddle points for the two integrals, and the product of the critical values is exponentially small or large. If $c$ is chosen so that the critical points coincide, ${ }^{*}$ then the product of the critical values is 1 and the product of the operators has nontrivial scaling.

To determine $c$, let $\sigma(z)=n^{-1} \log \psi(z)$, so that

$$
\sigma^{\prime}(z)=\frac{2 \alpha \tau}{1-\alpha^{2} z^{2}}+\frac{2 \alpha}{z^{2}-\alpha^{2}}-\frac{c}{z} .
$$

If we eliminate $c$ from $\sigma^{\prime}(z)=\sigma^{\prime \prime}(z)=0$, we obtain

$$
\frac{\tau\left(1+\alpha^{2} z^{2}\right)}{\left(1-\alpha^{2} z^{2}\right)^{2}}-\frac{\alpha^{2}+z^{2}}{\left(z^{2}-\alpha^{2}\right)^{2}}=0 .
$$

The function on the left is strictly increasing from $-\infty$ to $+\infty$ on the interval $\left(\alpha, \alpha^{-1}\right)$. It follows that there is a unique point $z_{0}$ in this interval where the function vanishes. This is our saddle point, and we set

$$
c=2 \alpha z_{0}\left(\frac{\tau}{1-\alpha^{2} z_{0}^{2}}+\frac{1}{z_{0}^{2}-\alpha^{2}}\right)>0 .
$$

From the behavior of $\sigma^{\prime}(z)$ for large negative $z$ and near $-\alpha^{-1}, \alpha$, and zero, we see that $\sigma^{\prime}$ has a zero in $\left(-\infty,-\alpha^{-1}\right)$ and a zero in $(-\alpha, 0)$. Since it has a double zero at $z_{0}$, this accounts for all four of its finite zeros. Since $\sigma^{\prime}(z)$ tends to $+\infty$ at the endpoints of $\left(\alpha, \alpha^{-1}\right)$, it follows that it is positive everywhere there except at $z_{0}$, and this implies that $\sigma^{\prime \prime \prime}\left(z_{0}\right)>0$. (Well, this only shows that $\sigma^{\prime \prime \prime}\left(z_{0}\right) \geq 0$. We find in Section 6.6 an explicit expression for $\sigma^{\prime \prime \prime}\left(z_{0}\right)$ in terms of $z_{0}$, from which it is clear that it is positive.)

The two steepest descent curves, which we call $\Gamma^{+}$for the first integral and $\Gamma^{-}$ for the second, together form a single contour. The first emanates from $z_{0}$ at angles $\pm \pi / 3$ with branches going to $\infty$ in two directions. The second emanates from $z_{0}$ at angles $\pm 2 \pi / 3$ and closes at $z=0$ (see Figure 2 ).

It is convenient to replace $\ell^{2}([h, \infty))$ by $\ell^{2}\left(\mathbb{Z}_{+}\right)$, and to do that we change our meaning of the operator $P$. A $P$ appearing on the left is to be interpreted as $\Lambda^{h}$, 


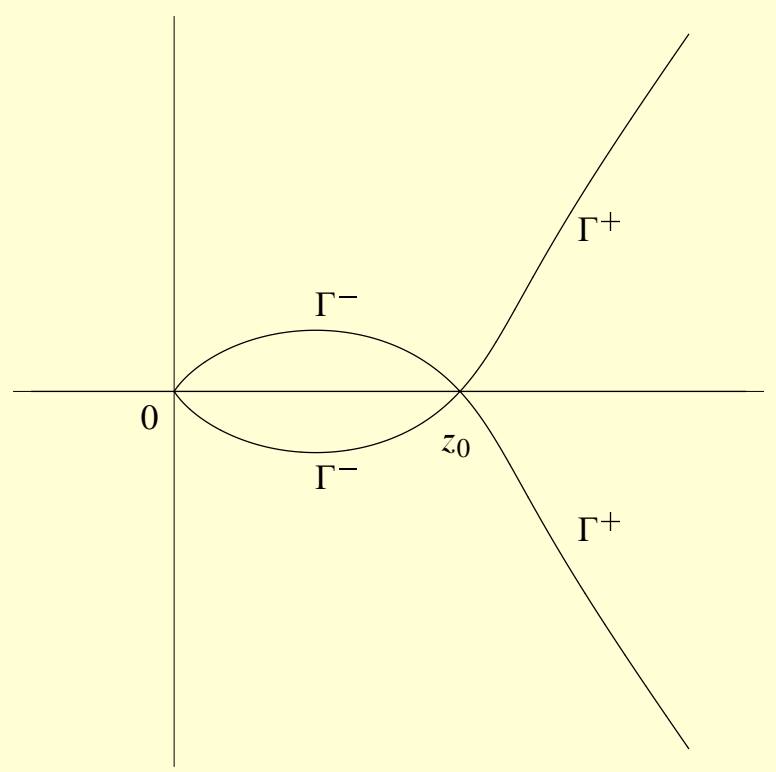

Figure 2. Steepest descent curves $\Gamma^{ \pm}$

and a $P$ appearing on the right is to be interpreted as $\Lambda^{* h}$. So, for example, the $i$ th component of $P T_{1} e$ is $\left(T_{1} e\right)_{h+i}$ and the $(i, j)$-entry of $P H_{1} H_{2}$ is $\left(H_{1} H_{2}\right)_{h+i, h+j}$. With these reinterpretations of $P$, the operators in (6.22) all act on $\ell^{2}\left(\mathbb{Z}_{+}\right)$and the determinant has not changed. (Recall that $h=c n+n^{1 / 3} s$.)

Let $D$ be the diagonal matrix with $i$ th diagonal element equal to $\psi\left(z_{0}\right)^{-1} z_{0}^{n^{1 / 3} s+i}$, and multiply (6.22) by $\left(\begin{array}{cc}D & 0 \\ 0 & D^{-1}\end{array}\right)$ on the left and by $\left(\begin{array}{cc}D^{-1} & 0 \\ 0 & D\end{array}\right)$ on the right. This does not affect the determinant. (The reason is that all the operators in (6.22) have $(i, j)$ entry $O\left(r^{i+j}\right)$ for fixed $n$, where $r$ can be any number larger than $\alpha$, and $\alpha<z_{0}<$ $\alpha^{-1}$.) We show that after these multiplications the first operator scales to the the direct sum of two Airy operators, * the second operator has trace norm $o(1)$, and the vectors in the sum all have norm $o(1)$.

*If a matrix acting on $\ell^{2}\left(\mathbb{Z}_{+}\right)$has entries $M(i, j)$ and if the kernel $n^{1 / 3} M\left(\left[n^{1 / 3} x\right],\left[n^{1 / 3} y\right]\right)$ acting on $L^{2}(0, \infty)$, which is unitarily equivalent to the matrix operator, converges in trace norm to a limiting kernel, then we say that the matrix scales in trace norm to the limiting kernel. The Fredholm determinant of $M(i, j)$ then converges to the Fredholm determinant of the limiting kernel. This is what happens here, with the limiting kernel being the direct sum of two Airy kernels. 


\subsubsection{The first operator in (6.22)}

The upper left corner of the first operator becomes, aside from the identity operator, $D P H_{1} H_{2} P D^{-1}$. We write this as

$$
\left(D P H_{1} D_{0}\right)\left(D_{0}^{-1} H_{2} P D^{-1}\right),
$$

where $D_{0}$ is the diagonal matrix with $i$ th diagonal element equal to $z_{0}^{i}$, and we scale each factor. We have

$$
\begin{aligned}
z_{0}^{n^{1 / 3} s+i+j}\left(P H_{1}\right)_{i, j} & =\frac{z_{0}^{n^{1 / 3} s+i+j}}{2 \pi \mathrm{i}} \int\left(\frac{1+\alpha z}{1-\alpha z}\right)^{m}\left(\frac{z-\alpha}{z+\alpha}\right)^{n} z^{-h-i-j-2} \mathrm{~d} z \\
& =\frac{z_{0}^{n^{1 / 3} s+i+j}}{2 \pi \mathrm{i}} \int \psi(z) z^{-n^{1 / 3} s-i-j} \frac{\mathrm{d} z}{z^{2}}
\end{aligned}
$$

The main fact is the following. Define

$$
\psi(z, \gamma)=\left(\frac{1+\alpha z}{1-\alpha z}\right)^{m}\left(\frac{z-\alpha}{z+\alpha}\right)^{n} z^{-\gamma n},
$$

and set

$$
I_{n}(x)=\frac{1}{2 \pi \mathrm{i}} \int \psi\left(z, c+n^{-2 / 3} x\right) \frac{\mathrm{d} z}{z^{2}},
$$

the contour being the unit circle. Then

$$
\psi\left(z_{0}\right)^{-1} z_{0}^{n^{1 / 3} x} n^{1 / 3}\left|I_{n}(x)\right| \leq e^{-\delta x},
$$

valid for some $\delta$ and all $n$ if $x$ is bounded from below, and

$$
\lim _{n \rightarrow \infty} \psi\left(z_{0}\right)^{-1} z_{0}^{n^{1 / 3} x} n^{1 / 3} I_{n}(x)=z_{0}^{-1} g \operatorname{Ai}(g x)
$$

pointwise, with $g$ the constant given by (6.30). (The limit in (6.27) is uniform for $x$ in a bounded set.)

We first show that (6.26) holds if $n^{1 / 3} x>\eta n$ for some $\eta>0$. For this we set $\gamma=c+n^{-2 / 3} x$ so that $\gamma-c>\eta$, write our main integrand as $\psi(z, \gamma)$, and do steepest descent. With

$$
\sigma(z, \gamma)=n^{-1} \log \psi(z, \gamma)=\sigma(z)-(\gamma-c) \log z,
$$

our saddle points $z_{\gamma}^{ \pm}$(there are two of them when $\gamma>c$ ) are solutions of $\sigma^{\prime}\left(z_{\gamma}^{ \pm}, \gamma\right)=$ 0 . Differentiating this with respect to $\gamma$ gives

$$
\sigma^{\prime \prime}\left(z_{\gamma}^{ \pm}, \gamma\right) \frac{d z_{\gamma}^{ \pm}}{d \gamma}-\frac{1}{z_{\gamma}^{ \pm}}=0 .
$$


Since $\sigma^{\prime \prime}\left(z_{\gamma}^{ \pm}, \gamma\right) \neq 0$ (since $c$ is the only value of $\gamma$ for which there is a double saddle point), we find that if $z_{\gamma}^{ \pm}$are chosen so that $z_{\gamma}^{+}>z_{\gamma}^{-}$for $\gamma$ near $c$, then $z_{\gamma}^{+}$increases always as $\gamma$ increases and $z_{\gamma}^{-}$decreases, and $\sigma_{1}^{\prime \prime}\left(z_{\gamma}^{+}, \gamma\right)>0$ and $\sigma_{1}^{\prime \prime}\left(z_{\gamma}^{-}, \gamma\right)<0$. In particular, $z_{\gamma}^{+}$is the saddle point we take for our steepest descent, and $z_{\gamma}^{+}>z_{0}$ when $\gamma>c$ since $z_{c}^{+}=z_{0}$. For the critical value, we have to see how $\sigma\left(z_{\gamma}^{+}, \gamma\right)$ behaves as a function of $\gamma$. From (6.28) and the fact $\sigma^{\prime}\left(z_{\gamma}^{ \pm}, \gamma\right)=0$, we obtain

$$
\frac{d \sigma\left(z_{\gamma}^{ \pm}, \gamma\right)}{d \gamma}=-\log z_{\gamma}^{ \pm},
$$

and so

$$
\frac{d}{d \gamma}\left[\sigma\left(z_{\gamma}^{ \pm}, \gamma\right)+\gamma \log z_{0}\right]=\log \frac{z_{0}}{z_{\gamma}^{ \pm}} .
$$

Since $z_{\gamma}^{+}$is an increasing function of $\gamma$, this shows that $\sigma\left(z_{\gamma}^{+}, \gamma\right)+\gamma \log z_{0}$ is a decreasing concave function of $\gamma$. It follows that for some $\delta>0$,

$$
\sigma\left(z_{\gamma}^{+}, \gamma\right)+\gamma \log z_{0}<\sigma\left(z_{0}\right)+c \log z_{0}-\delta(\gamma-c)
$$

for $\gamma \geq c+\eta$. Thus for these $\gamma$,

$$
\psi\left(z_{0}\right)^{-1} z_{0}^{n(\gamma-c)} \psi\left(z_{\gamma}^{+}, \gamma\right) \leq \mathrm{e}^{-\delta n(\gamma-c)} .
$$

Since $|\psi(z, \gamma)|$ achieves its maximum on the steepest descent contour at $z_{\gamma}$ and since the contour is bounded away from zero (this follows from the fact that $z_{\gamma} \rightarrow \alpha$ as $\gamma \rightarrow \infty)$, we see that in this case $I_{n}(x)$ is at most a constant times $\psi\left(z_{\gamma}^{+}, \gamma\right)$, where $\gamma=c+n^{-2 / 3} x$. Hence

$$
\psi\left(z_{0}\right)^{-1} z_{0}^{n^{1 / 3} x}\left|I_{n}(x)\right|=O\left(\mathrm{e}^{-\delta n^{1 / 3} x}\right)
$$

when $n^{1 / 3} x>\eta n$. This is an even better estimate than (6.26).

Since we have shown that (6.26) holds if $n^{1 / 3} x>\eta n$, where $\eta$ can be as small as we please, we may assume $n^{1 / 3} x=o(n)$ when $x>0$.

Write

$$
I_{n}(x)=\frac{1}{2 \pi \mathrm{i}} \int \psi(z) z^{-n^{1 / 3} x} \frac{\mathrm{d} z}{z^{2}},
$$

and use the steepest descent curve $\Gamma^{+}$. It emanates from $z_{0}$ at angles $\pm \pi / 3$. Clearly it is bounded away from zero. Choose $\varepsilon$ small, and let

$$
\Gamma^{(1)}=\left\{z \in \Gamma^{+}:\left|z-z_{0}\right|>\varepsilon\right\} \quad \text { and } \quad \Gamma^{(2)}=\left\{z \in \Gamma^{+}:\left|z-z_{0}\right|<\varepsilon\right\}
$$

with corresponding $I_{n}^{(1)}(x)$ and $I_{n}^{(2)}(x)$. We show that both of these satisfy the uniform estimate (6.26) and

$$
\lim _{n \rightarrow \infty} \psi\left(z_{0}\right)^{-1} n^{1 / 3} I_{n}^{(1)}(x)=0
$$


and

$$
\lim _{n \rightarrow \infty} \psi\left(z_{0}\right)^{-1} z_{0}^{n^{1 / 3} x} n^{1 / 3} I_{n}^{(2)}(x)=z_{0}^{-1} g \operatorname{Ai}(g x) .
$$

Consider $I_{n}^{(1)}(x)$ first. Since $\Re \sigma(z)$ is strictly decreasing as we move away from $z_{0}$ on $\Gamma$, we know that $|\psi(z)|<\psi\left(z_{0}\right) \mathrm{e}^{-\delta n}$ on $\Gamma^{(1)}$ for some $\delta>0$. The assertions follow from this since we are in the case $n^{1 / 3} x=o(n)$. (This also holds also for $x<0$ since $x$ is bounded below.)

Making the variable change $z \rightarrow z_{0}(1+\xi)$, we can write, since $z=z_{0} e^{\xi(1+O(\xi))}$ near $z=z_{0}$,

$I_{n}^{(2)}(x)=\psi\left(z_{0}\right) z_{0}^{-n^{1 / 3} x} \frac{1}{2} \pi \mathrm{i} \int_{|\xi|<\varepsilon / z_{0}} \mathrm{e}^{n b z_{0}^{3} \xi^{3}(1+O(\xi))-n^{1 / 3} x \xi(1+O(\xi))}\left(z_{0}^{-1}+O(\xi)\right) \mathrm{d} \xi$,

where $b=\sigma^{\prime \prime \prime}\left(z_{0}\right) / 6$. The path of integration here is the portion of the contour $\Gamma^{(2)}$ satisfying the indicated inequality. It consists of two little arcs emanating from $\xi=0$ tangent to the line segments making angles $\pm \pi / 3$ with the positive axis. If $\varepsilon$ is small enough and we replace the integral by the line segments themselves, we introduce an error of the form $O\left(e^{-\delta n}\right)$ with a different $\delta$ since $n^{1 / 3} x=o(n)$. With the variable change $\xi \rightarrow n^{-1 / 3} \xi$, we obtain

$$
\begin{aligned}
& \psi\left(z_{0}\right)^{-1} z_{0}^{n^{1 / 3} x} n^{1 / 3} I_{n}^{(2)}(x) \\
& =\frac{1}{2 \pi \mathrm{i}} \int_{|\xi|<n^{1 / 3} \varepsilon} \mathrm{e}^{b z_{0}^{3} \xi^{3}\left(1+O\left(n^{-1 / 3} \xi\right)\right)-x \xi\left(1+O\left(n^{-1 / 3} \xi\right)\right)}\left(z_{0}^{-1}+O\left(n^{-1 / 3} \xi\right)\right) \mathrm{d} \xi \\
& \quad+O\left(e^{-\delta n}\right),
\end{aligned}
$$

where now the integration is taken over line segments of length of the order $n^{1 / 3}$.

On the path of integration, we have $\Re\left(\xi^{3}\right) \leq-\delta|\xi|^{3}$ and $\Re(\xi) \geq \delta|\xi|$ for some $\delta>0$, and so for the above we have an estimate of the form

$$
\int_{0}^{\infty} \mathrm{e}^{-\delta\left(t^{3}-x t\right)} \mathrm{d} t+O\left(e^{-\delta n}\right)
$$

Since $n \gg x$, this gives the required uniform bound. The limit of the integral, with its factor $1 / 2 \pi \mathrm{i}$, equals $z_{0}^{-1} g \operatorname{Ai}(g x)$, where

$$
g=\left(3 b z_{0}^{3}\right)^{-1 / 3}=z_{0}^{-1}\left(\frac{2}{\sigma^{\prime \prime \prime}\left(z_{0}\right)}\right)^{1 / 3} .
$$

That the limit is as stated follows by taking the limit under the integral sign, which is justified by dominated convergence.

To obtain the scaling of the matrix $D P H_{1} D_{0}$, we need only observe that by (6.25),

$$
\begin{aligned}
& n^{1 / 3}\left(D P H_{1} D_{0}\right)_{\left[n^{1 / 3} x\right],\left[n^{1 / 3} y\right]} \\
& \quad=\psi\left(z_{0}\right)^{-1} z_{0}^{n^{1 / 3} s+\left[n^{1 / 3} x\right]+\left[n^{1 / 3} x\right]} n^{1 / 3} I_{n}\left(s+n^{-1 / 3}\left(\left[n^{1 / 3} x\right]+\left[n^{1 / 3} y\right]\right)\right) .
\end{aligned}
$$


It follows from (6.26) and (6.27) that this kernel on $(0, \infty)$ converges in HilbertSchmidt norm to $z_{0}^{-1} g \operatorname{Ai}(g(s+x+y))$.

To scale $D_{0}^{-1} H_{2} P D^{-1}$, we write

$$
z_{0}^{-n^{1 / 3} s-i-j}\left(P H_{2}\right)_{i, j}=\frac{z_{0}^{-n^{1 / 3} s-i-j}}{2 \pi \mathrm{i}} \int\left(\frac{1+\alpha z}{1-\alpha z}\right)^{n}\left(\frac{z-\alpha}{z+\alpha}\right)^{m} z^{-c n-n^{1 / 3} s-i-j-2} \mathrm{~d} z .
$$

If we make the substitution $z \rightarrow z^{-1}$, this becomes

$$
\frac{z_{0}^{-n^{1 / 3} s-i-j}}{2 \pi \mathrm{i}} \int \psi(z)^{-1} z^{n^{1 / 3} s+i+j} \mathrm{~d} z .
$$

This is completely analogous to (6.25). To use the analogous argument we mention only that we use (6.29) with the minus signs to see that both $-\sigma\left(z_{\gamma}^{-}, \gamma\right)-\gamma \log z_{0}$ and its derivative are decreasing functions of $\gamma$. The steepest descent curve now is $\Gamma^{-}$. We need not go through the details again. We find that

$$
n^{1 / 3}\left(D_{0}^{-1} P H_{1} D^{-1}\right)_{\left[n^{1 / 3} x\right],\left[n^{1 / 3} y\right]}
$$

converges in Hilbert-Schmidt norm to $z_{0} g \operatorname{Ai}(g(s+x+y))$. Hence

$$
n^{1 / 3}\left(D P H_{1} H_{2} P D^{-1}\right)_{\left[n^{1 / 3} x\right],\left[n^{1 / 3} y\right]}
$$

converges in trace norm to $g K_{\text {Airy }}(g(s+x), g(x+y))$ on $L^{2}(0, \infty)$.

By taking transposes, we see that $D^{-1} \mathrm{PH}_{2} \mathrm{H}_{1} P D^{-1}$ has the same scaling limit, which takes care of the lower right corner of the first operator in (6.22).

\subsubsection{The second operator in (6.22)}

Next, we have to look at

$$
D P\left(I-H_{1} H_{2}\right) L_{x} \Lambda P D \quad \text { and } \quad D^{-1} P\left(I-H_{2} H_{1}\right) L_{y} \Lambda P D^{-1} .
$$

We find, using (6.14),

$$
\begin{aligned}
& z_{0}^{2 n^{1 / 3} s+i+j}\left(\left(I-H_{1} H_{2}\right) L_{x} \Lambda\right)_{h+i, h+j} \\
&=\left(\frac{1}{2 \pi \mathrm{i}}\right)^{2} \iint\left(\frac{1+\alpha z}{1-\alpha z}\right)^{m}\left(\frac{z-\alpha}{z+\alpha}\right)^{n}\left(\frac{1+\alpha \zeta}{1-\alpha \zeta}\right)^{n}\left(\frac{\zeta-\alpha}{\zeta+\alpha}\right)^{m} \\
& \times z_{0}^{2 n^{1 / 3} s+i+j} \frac{z^{-h-i-1} \zeta^{h+j-1}}{z \zeta-1} \mathrm{~d} z \mathrm{~d} \zeta(-1)^{h+j}
\end{aligned}
$$

After the substitution of $\zeta \rightarrow-\zeta^{-1}$, this becomes

$$
\left(\frac{1}{2 \pi \mathrm{i}}\right)^{2} \iint \psi(z) \psi(\zeta)\left(\frac{z}{z_{0}}\right)^{-n^{1 / 3} s-i}\left(\frac{\zeta}{z_{0}}\right)^{-n^{1 / 3} s-j} \frac{\mathrm{d} z \mathrm{~d} \zeta}{z(z+\zeta)} .
$$


The integrals here are initially taken over circles close to the unit circle, with $|z|>|\zeta|$. We first deform the $\zeta$-contour to $\Gamma^{+}$while always having $z+\zeta$ nonzero. Then if we deform the $z$-contour, we pass through a pole at $\zeta=-z$ for every $z \in \Gamma^{+}$. The residue at the pole equals a constant times $z^{-2 h-i-j-2}$, and integrating this over $\Gamma^{+}$gives zero. So both integrals may be taken over $\Gamma^{+}$. Since now the denominator does not vanish at $z=\zeta=z_{0}$, the same sort of argument we already gave shows that this operator equals a constant times $\psi\left(z_{0}\right)^{2} n^{-1 / 3}$ times an operator that scales to the trace class operator $g \operatorname{Ai}(g(s+x)) \otimes g \operatorname{Ai}(g(s+y))$. In particular, its trace norm is $O\left(\psi\left(z_{0}\right)^{2} n^{-1 / 3}\right)$. This shows that $D P\left(I-H_{1} H_{2}\right) L_{x} \Lambda P D$ has trace norm $O\left(n^{-1 / 3}\right)$, and a similar argument applies to $D^{-1} P\left(I-H_{2} H_{1}\right) L_{y} \Lambda P D^{-1}$.

\subsubsection{The last operator in (6.22)}

Finally, we consider the vectors $P a_{i}$ and $P b_{j}^{\prime}$ and look at their constituents $P T_{1} e$, $P T_{2} e, H_{1} H_{2} e$, and $H_{2} H_{1} e$. We show that

$$
\begin{aligned}
& \left\|D P T_{1} e\right\|=O\left(n^{-1 / 6}\right) \quad \text { and } \quad \mid D^{-1} P T_{2} e \|=O\left(n^{-1 / 6}\right), \\
& \left\|D H_{1} H_{2} e\right\|=O\left(n^{-2 / 3}\right) \quad \text { and } \quad\left\|D^{-1} H_{2} H_{1} e\right\|=O\left(n^{-2 / 3}\right) \text {. }
\end{aligned}
$$

For the first, we have

$$
\left(T_{1} e\right)_{h+i}=\frac{1}{2} \pi \mathrm{i} \int \psi(z) z^{-n^{1 / 3} s-i-1} \mathrm{~d} z=I_{n}\left(s+n^{-1 / 3}(i-1)\right),
$$

and from (6.26) and (6.27) we deduce now that the function

$$
n^{1 / 3}\left(D P T_{1} e\right)_{\left[n^{1 / 3} x\right]}
$$

converges in $L^{2}(0, \infty)$. In particular, its norm is $O(1)$. But then

$$
\left\|\left\{\left(D P T_{1} e\right)\right\}_{i}\right\|_{\ell^{2}}=n^{1 / 6}\left\|\left(D P T_{1} e\right)_{\left[n^{1 / 3} x\right]}\right\|_{L^{2}}=O\left(n^{-1 / 6}\right) .
$$

Similarly, $\left\|D^{-1} P T_{2} e\right\|=O\left(n^{-1 / 6}\right)$.

For $\mathrm{DH}_{1} \mathrm{H}_{2} e$, we have from (6.21),

$$
\begin{aligned}
z_{0}^{n^{1 / 3} s+i} & \left(H_{1} H_{2} e\right)_{h+i} \\
= & \left(\frac{1}{2 \pi \mathrm{i}}\right)^{2} \iint\left(\frac{1+\alpha z}{1-\alpha z}\right)^{m}\left(\frac{z-\alpha}{z+\alpha}\right)^{n}\left(\frac{1+\alpha \zeta}{1-\alpha \zeta}\right)^{n}\left(\frac{\zeta-\alpha}{\zeta+\alpha}\right)^{m} \\
& \times z^{-h-i-1} z_{0}^{n^{1 / 3} s+i} \zeta^{-1} \frac{\mathrm{d} z \mathrm{~d} \zeta}{z \zeta-1},
\end{aligned}
$$

and with the substitution $\zeta \rightarrow \zeta^{-1}$, this becomes

$$
\left(\frac{1}{2 \pi \mathrm{i}}\right)^{2} \iint \psi(z)\left(\frac{\zeta+\alpha}{\zeta-\alpha}\right)^{n}\left(\frac{1-\alpha \zeta}{1+\alpha \zeta}\right)^{m} z^{-n^{1 / 3} s-i-1} z_{0}^{n^{1 / 3} s+i} \frac{\mathrm{d} z \mathrm{~d} \zeta}{z-\zeta} .
$$


As before, the integrals here are initially taken over circles close to the unit circle, with $|z|>|\zeta|$. Now we want to deform the $z$-contour to $\Gamma^{+}$and the $\zeta$-contour to its steepest descent contour $C$, a curve passing through the saddle points $\pm i \beta^{-1}$ and closing at $-\alpha$ and $\alpha^{-1}$.

To do this, we show first that, except for $z_{0}$, all points of $\Gamma^{+}$satisfy $|z|>z_{0}$. This follows if we can show that on the circle $z=z_{0} \mathrm{e}^{\mathrm{i} \theta}$ the absolute minimum of

$$
\log \left|\left(\frac{1+\alpha z}{1-\alpha z}\right)^{\tau}\left(\frac{z-\alpha}{z+\alpha}\right)\right|
$$

occurs at $\theta=0$. (For then $|\psi(z)|$ is larger than $\psi\left(z_{0}\right)$ everywhere on the circle except for $z=z_{0}$, so no other point on the circle could be on $\Gamma^{+}$. Locally, $\Gamma^{+}$is outside the circle, and so it has to be everywhere outside.) Using

$$
\frac{d}{d \theta}=\mathrm{i} z \frac{d}{d z}
$$

we find that the derivative with respect to $\theta$ of (6.32) equals $-2 \alpha$ times the imaginary part of

$$
\frac{\tau}{z^{-1}-\alpha^{2} z}+\frac{1}{z-\alpha^{2} z^{-1}}=\frac{\left(\tau-\alpha^{2}\right) z+\left(1-\tau \alpha^{2}\right) z^{-1}}{\left(z^{-1}-\alpha^{2} z\right)\left(z-\alpha^{2} z^{-1}\right)} \text {. }
$$

This vanishes exactly when the imaginary part of

$$
\left(\left(\tau-\alpha^{2}\right) z+\left(1-\alpha^{2}\right) z^{-1}\right)\left(\bar{z}^{-1}-\alpha^{2} \bar{z}\right)\left(\bar{z}-\alpha^{2} \bar{z}^{-1}\right)
$$

does. This is a trigonometric polynomial in $\theta$ of degree 3. It is an odd function of $\theta$ and so is of the form $\sin \theta$ times a polynomial of degree two in $\cos \theta$. Since it has at least a double zero at $\theta=0$ (by the choice of $c$ and $z_{0}$ ), the polynomial must have a factor $\cos \theta-1$. Since it is an odd function of $z$, it must also have a double zero at $\theta=\pi$, so there must also be a factor $\cos \theta+1$. Thus it must be equal to a constant times $\sin \theta\left(\cos ^{2} \theta-1\right)$. In particular, there can be no other zeros. Thus (6.32), which we know has a local minimum on the circle at $z=z_{0}$, must have its absolute minimum there (and its absolute maximum at $z=-z_{0}$ ). Thus, as claimed, all points of $\Gamma^{+}$except for $z_{0}$ satisfy $|z|>z_{0}$.

In particular, all points of $\Gamma^{+}$satisfy $|z| \geq z_{0}$. Since $z_{0}>\alpha$, we can first take the integrals in (6.31) over the circles $|z|=z_{0}$ and $|\zeta|=\alpha+\varepsilon$ with $\varepsilon$ small and positive. Then we can deform the $z$-contour to $\Gamma^{+}$without crossing the circle $|\zeta|=\alpha+\varepsilon$. Next, we want to deform the $\zeta$-contour to $C$. This curve closes on the right at $\alpha^{-1}$, and so since $z_{0}<\alpha^{-1}$, it intersects $\Gamma^{+}$at two points, $z^{\prime}$ and $z^{\prime \prime}$, say. (In principle there could be finitely many other points; the following argument could be easily modified in this case.) Hence upon deforming the $\zeta$-contour to $C$, we pass through a pole for those $z$ on the arc of $\Gamma^{+}$passing through $z=z_{0}$ with end points $z^{\prime}$ and $z^{\prime \prime}$. For each 
$z$ on this arc, the residue at $\zeta=z$ equals $z^{-h-i-1} z_{0}^{n^{1 / 3} s+i}$, and then integrating with respect to $z$ gives

$$
z_{0}^{n^{1 / 3} s}(h+i)^{-1}\left[\frac{z_{0}^{i}}{z^{\prime \prime h+i}}-\frac{z_{0}^{i}}{z^{\prime h+i}}\right] .
$$

We claim that $\psi\left(z_{0}\right)^{-1}$ (which is the factor contained in $D$ ) times this vector is exponentially small, that is, $O\left(\mathrm{e}^{-\delta n}\right)$ for some $\delta>0$.

Because all points of $\Gamma^{+}$except $z_{0}$ satisfy $|z|>z_{0}$, the vectors $\left\{\left(z_{0} / z^{\prime \prime}\right)^{i}\right\}$ and $\left\{\left(z_{0} / z^{\prime}\right)^{i}\right\}$ belong to $\ell^{2}\left(\mathbb{Z}_{+}\right)$. So we need only show that $\psi\left(z_{0}\right)^{-1}\left|z^{\prime}\right|^{-h}$ is exponentially small (and the same for $z^{\prime \prime}$ ). In fact, on the part of $C$ in the right half-plane,

$$
\left|\left(\frac{z+\alpha}{z-\alpha}\right)\left(\frac{1-\alpha z}{1+\alpha z}\right)^{\tau}\right|
$$

is at most 1 and is strictly less than 1 outside a neighborhood of the critical points $\pm i \beta$. This shows that $\left|z^{\prime}\right|^{-c} \leq(1-\delta)\left|\psi\left(z^{\prime}\right)\right|^{1 / n}$ for some $\delta>0$. Since $|\psi(z)|<\left|\psi\left(z_{0}\right)\right|$ on $\Gamma^{+}$, this shows that $\left|z^{\prime}\right|^{-c} \leq(1-\delta)\left|\psi\left(z_{0}\right)\right|^{1 / n}$, and it follows that $\psi\left(z_{0}\right)^{-1}\left|z^{\prime}\right|^{-h}$ is exponentially small.

We can now say that, with error $\psi\left(z_{0}\right)^{2}$ times an exponentially small quantity, the square of the norm of the vector (6.31) equals a quadruple integral in which every term in the integrand except $z^{-h-i-1} z_{0}^{i}$ has an analogous term with variables $z^{\prime}, \zeta^{\prime}$, and the $z^{-h-i-1} z_{0}^{i}$-term becomes $\left(z z^{\prime}\right)^{-h} /\left(z z^{\prime}-z_{0}^{2}\right)$. For the $z$ - and $z^{\prime}$-integrals we integrate over $\Gamma^{+}$, and for the $\zeta$ - and $\zeta^{\prime}$-integrals we integrate over $C$. (Here we use again the fact that $|z|,\left|z^{\prime}\right|>z_{0}$, so we can sum under the integral signs.) The $\left(\zeta, \zeta^{\prime}\right)$ integrals contribute $O\left(n^{-1}\right)$, while the $\left(z, z^{\prime}\right)$-integrals contribute $O\left(\psi\left(z_{0}\right)^{2} n^{-1 / 3}\right)$. Thus $\left\|D P H_{1} H_{2} e\right\|^{2}=O\left(n^{-4 / 3}\right)$.*

For $H_{2} H_{1} e$ we interchange $m$ and $n$ and make the variable change $z \rightarrow z^{-1}$ but not the variable change $\zeta \rightarrow \zeta^{-1}$. Thus

$$
\begin{aligned}
z_{0}^{-n^{1 / 3} s-i} & \left(H_{2} H_{1} e\right)_{h+i} \\
= & \left(\frac{1}{2 \pi \mathrm{i}}\right)^{2} \iint \psi(z)^{-1}\left(\frac{1+\alpha \zeta}{1-\alpha \zeta}\right)^{n}\left(\frac{\zeta-\alpha}{\zeta+\alpha}\right)^{m} z^{n^{1 / 3} s+i} z_{0}^{-n^{1 / 3} s-i} \frac{\mathrm{d} z \mathrm{~d} \zeta}{\zeta-z} .
\end{aligned}
$$

Here originally we must have $|\zeta|>|z|$ on the contours, and we want to deform them so that the $z$-contour becomes $\Gamma^{-}$and the $\zeta$-contour becomes what we again call $C$. Now in the deformation, we pass through a pole in the $\zeta$-integration for those $z$ on an $\operatorname{arc}$ of $\Gamma^{-}$passing through $z=z_{0}$ with end points that we again call $z^{\prime}$ and $z^{\prime \prime}$. The

*This was under the basic assumption $\alpha^{2}<\tau<\alpha^{-2}$. Otherwise, the $\zeta$ - and $\zeta^{\prime}$-integrals are only $O(1)$, with the result that $\left\|D H_{1} H_{2} e\right\|$ is only $O\left(n^{-1 / 6}\right)$, and the same holds for $\left\|D^{-1} H_{2} H_{1} e\right\|$. These are still good enough since $o(1)$ is all that is needed. 
residue equals $z^{h+i} z_{0}^{-n^{1 / 3} s-i}$, and integration with respect to $z$ gives

$$
z_{0}^{-n^{1 / 3} s}(h+i+1)^{-1}\left[\frac{z^{\prime \prime h+i+1}}{z_{0}^{i}}-\frac{z^{\prime h+i+1}}{z_{0}^{i}}\right] .
$$

This is completely analogous to what went before. Now $\left|z^{\prime}\right|,\left|z^{\prime \prime}\right|<z_{0}$ and $\psi\left(z_{0}\right)\left|z^{\prime}\right|^{h}$ is exponentially small. We continue as with $\mathrm{H}_{1} \mathrm{H}_{2} e$ and find that $\left\|D^{-1} \mathrm{PH}_{2} \mathrm{H}_{1} e\right\|^{2}=$ $O\left(n^{-4 / 3}\right)$.

If we go back to the forms of the $a_{i}$ and $b_{j}^{\prime}$ described earlier, we see that the vectors that arise after multiplying by the diagonal matrices $D$ and $D^{-1}$ are exactly the four whose norms we just estimated.

\subsection{Recapitulation}

We have shown that the matrix (6.22) acting on $\ell^{2}\left(\mathbb{Z}_{+}\right)$scales in trace norm to the kernel

$$
\left(\begin{array}{cc}
g K_{\text {Airy }}(g(s+x), g(x+y)) & 0 \\
0 & g K_{\text {Airy }}(g(s+x), g(x+y))
\end{array}\right)
$$

acting on $L^{2}(0, \infty)$. It follows that its Fredholm determinant converges to $F(g s)^{2}$. In view of (5.1) and (6.10), this establishes that for fixed $s$,

$$
\lim _{n \rightarrow \infty} \mathrm{P}_{\sigma}\left(L \leq c n+n^{1 / 3} s\right)=F_{2}(g s),
$$

where $c$ is determined by (6.23) and (6.24) and $g$ by (6.30). This gives the statement of the main theorem, where the constants $c_{1}(\alpha, \tau)$ and $c_{2}(\alpha, \tau)$ of the introduction are, respectively, $c$ and $g^{-1}$.

\subsection{Computation of $\sigma^{\prime \prime \prime}\left(z_{0}\right)$}

Think of $c$ and $z_{0}$ as functions of $\tau$, which they are. We have

$$
\sigma^{\prime \prime}(z)=4 \alpha z\left[\frac{\alpha^{2} \tau}{\left(1-\alpha^{2} z^{2}\right)^{2}}-\frac{1}{\left(z^{2}-\alpha^{2}\right)^{2}}\right]+\frac{c}{z^{2}} .
$$

Differentiating the identity $0=\sigma^{\prime \prime}\left(z_{0}\right)$ with respect to $\tau$ and using the above gives

$$
0=\sigma^{\prime \prime \prime}\left(z_{0}\right) z_{0}^{\prime}+\frac{4 \alpha^{3} z_{0}}{\left(1-\alpha^{2} z_{0}^{2}\right)^{2}}+\frac{c^{\prime}}{z_{0}^{2}},
$$

where $z_{0}^{\prime}$ and $c^{\prime}$ denote $d z_{0} / d \tau$ and $d c / d \tau$, respectively.

From (6.23) and (6.24) we find that $c$ is given in terms of $z_{0}$ by the relation

$$
c=\frac{4 \alpha\left(1-\alpha^{4}\right) z_{0}^{3}}{\left(1+\alpha^{2} z_{0}^{2}\right)\left(z_{0}^{2}-\alpha^{2}\right)^{2}} .
$$


We compute that

$$
\frac{d c}{d z_{0}}=-4 \frac{\alpha\left(1-\alpha^{4}\right) z_{0}^{2}\left(z_{0}^{2}+3 \alpha^{2}+3 \alpha^{2} z_{0}^{4}+\alpha^{4} z_{0}^{2}\right)}{\left(1+\alpha^{2} z_{0}^{2}\right)^{2}\left(z_{0}^{2}-\alpha^{2}\right)^{3}} .
$$

From (6.23), $\tau$ is given in terms of $z_{0}$ by

$$
\tau=\frac{\left(1-\alpha^{2} z_{0}^{2}\right)^{2}\left(\alpha^{2}+z_{0}^{2}\right)}{\left(1+\alpha^{2} z_{0}^{2}\right)\left(z_{0}^{2}-\alpha^{2}\right)^{2}} .
$$

We compute that

$$
\frac{d \tau}{d z_{0}}=-\frac{2\left(1-\alpha^{4}\right) z_{0}\left(1-\alpha^{2} z_{0}^{2}\right)\left(\alpha^{4} z_{0}^{2}+3 \alpha^{2} z_{0}^{4}+3 \alpha^{2}+z_{0}^{2}\right)}{\left(z_{0}^{2}-\alpha^{2}\right)^{3}\left(1+\alpha^{2} z_{0}^{2}\right)^{2}} .
$$

We first solve (6.33) for $\sigma^{\prime \prime \prime}\left(z_{0}\right)$ in terms of $z_{0}^{\prime}$ and $c^{\prime}$ (and $z_{0}$ ). Then we use (6.34) and (6.35) and the relations $z_{0}^{\prime}=\left(d \tau / d z_{0}\right)^{-1}$ and $c^{\prime}=\left(d c / d z_{0}\right) z_{0}^{\prime}$. We find that

$$
\sigma^{\prime \prime \prime}\left(z_{0}\right)=4 \frac{\alpha\left(1-\alpha^{4}\right)\left[\left(1+\alpha^{4}\right) z_{0}^{2}+3 \alpha^{2}\left(1+z_{0}^{4}\right)\right]}{\left(1-\alpha^{4} z_{0}^{4}\right)\left(z_{0}^{2}-\alpha^{2}\right)^{3}} .
$$

This is positive since $0<\alpha<1$ and $\alpha<z_{0}<\alpha^{-1}$.

\section{Poisson limit of the shifted Schur measure}

For Schur measure there are two interesting limiting cases: the exponential limit and the Poisson limit. The exponential limit of shifted Schur measure is supported on standard shifted tableaux, and hence it is expressible in terms of $f_{s}^{\lambda}$ (recall (2.2)). The Poisson limit of shifted Schur measure yields a natural interpretation of the maximizing rule as a symmetry condition of the process.* Namely, if one sets

$$
m=n, \quad \alpha=\frac{t}{n}
$$

and takes $n \rightarrow \infty$, the percolation-type model described in $\S 3$ becomes the following. Consider two Poisson processes of rate $t^{2}$, both in the (same) square $[0,1] \times[0,1]$. Hence one can imagine two types of points, marked and unmarked, in the square. Now with probability one, no two points, whether marked or unmarked, have the same $x$ or $y$ coordinates, and hence the strictly increasing conditions (2) and (3) of $\S 3$ for the rule of the maximizing path are not necessary. Therefore we do not need to distinguish the unmarked and marked points. Since the union of two Poisson processes has the rate $2 t^{2}$, the resulting process is as follows. In the square $[0,1] \times[0,1]$, select Poisson points of rate $2 t^{2}$. Then take the longest path, starting from the lower right corner 
$(1,0)$, that follows an up/left path, turns in direction once and only once, and follows an up/right path ending at the upper right corner $(1,1)$.

It is clear that this length is also equal to the following symmetric version. Take a realization of the Poisson process. Take the mirror image of the points about the left side. Adjoin the mirror image on the left and the original points on the right. Hence the resulting rectangle has sides of lengths 2 and 1, and there are twice as many points of the original configuration that are symmetric about the center vertical line. The (usual) longest up/right path from the left lower corner to the right upper corner is precisely the longest maximizing path from the lower right corner to the upper right corner in the above description.

The (formal) limit $m=n \rightarrow \infty$ with $\alpha=t / n$ ( $t$ fixed) in the main theorem of $\S 1$ is

$$
\lim \mathrm{P}\left(\frac{L-4 t}{(2 t)^{1 / 3}}<s\right)=F_{2}(s) .
$$

The consequence is that the vertically symmetric Poisson process has the same fluctuation as the usual Poisson process with no symmetry condition. Also, the scaling in the above result is consistent with this intuition. There are Poisson points of rate $4 t^{2}$ (double of $2 t^{2}$ ) in the square of sides 2 and 1. For such the case the limit (7.1) is also valid for the case of no symmetry condition.

Acknowledgments. The authors thank Richard Stanley and Sergey Fomin for useful early discussions concerning the RSK correspondence and the referee for the remarks of $\S 7$.

\section{References}

[AM] M. ADLER and P. VAN MOERBEKE, Integrals over classical groups, random permutations, Toda and Toeplitz lattices, Comm. Pure Appl. Math. 54 (2001), 153-205. MR 1794352173

[AD] D. ALDOUS and P. DIACONIS, Longest increasing subsequences: From patience sorting to the Baik-Deift-Johansson theorem, Bull. Amer. Math. Soc. (N.S.) 36 (1999), 413-432. MR 1694204180

[BDJ] J. BAIK, P. DEIFT, and K. JOHANSSON, On the distribution of the length of the longest increasing subsequence of random permutations, J. Amer. Math. Soc. 12 (1999), 1119-1178. MR 1682248172

[BR1] J. BAIK and E. M. RAINS, Limiting distributions for a polynuclear growth model with external sources, J. Statist. Phys. 100 (2000), 523 - 541. MR 1788477173

[BR2] - The asymptotics of monotone subsequences of involutions, Duke Math. J. 109 (2001), 205-281. MR 1845180173

[BR3] - "Symmetrized random permutations" in Random Matrix Models and Their Applications, ed. P. Bleher and A. Its, Math. Sci. Res. Inst. Publ. 40, Cambridge Univ. Press, Cambridge, 2001, 1-19. MR 1842780173 
[BW] E. L. BASOR and H. WIDOM, On a Toeplitz determinant identity of Borodin and Okounkov, Integral Equations Operator Theory 37 (2000), 397 -401. MR 1780119 172

[BO] A. BORODIN and A. OKOUNKOV, A Fredholm determinant formula for Toeplitz determinants, Integral Equations Operator Theory 37 (2000), 386-396. MR 1700118172

[BOO] A. BORODIN, A. OKOUNKOV, and G. OLSHANSKI, Asymptotics of Plancherel measures for symmetric groups, J. Amer. Math. Soc. 13 (2000), 481 -515. MR 1758751173

[BS] A. BÖTTCHER and B. SILBERMANN, Analysis of Toeplitz Operators, Springer, Berlin, 1990. MR 1071374172

[DZ] P. A. DEIFT and X. ZHOU, A steepest descent method for oscillatory Riemann-Hilbert problems: Asymptotics for the MKdV equation, Ann. of Math. (2) 137 (1993), 295-368. MR 1207209173

[Ge] I. M. GESSEL, Symmetric functions and P-recursiveness, J. Combin. Theory Ser. A 53 (1990), 257-285. MR 1041448 172, 180

[GTW1] J. GRAVNER, C. A. TRACY, and H. WIDOM, Limit theorems for height fluctuations in a class of discrete space and time growth models, J. Statist. Phys. 102 (2001), 1085-1132. MR 1830441173

[GTW2] - A growth model in a random environment, Ann. Probab. 30 (2002), 1340-1368. MR 1920110173

[HH] P. N. HOFFMAN and J. F. HUMPHREYS, Projective Representations of the Symmetric Groups: Q-Functions and Shifted Tableaux, Oxford Math. Monogr., Oxford Univ. Press, New York, 1992. MR 1205350 173, 174, 179

[IW] M. ISHIKAWA and M. WAKAYAMA, Minor summation formula of Pfaffians, Linear Multilinear Algebra 39 (1995), 285-305. MR 1365449 173, 180, 181

[ITW] A. R. ITS, C. A. TRACY, and H. WIDOM, "Random words, Toeplitz determinants, and integrable systems, I" in Random Matrix Models and Their Applications, Math. Sci. Res. Inst. Publ. 40, ed. P. Bleher and A. R. Its, Cambridge Univ. Press, Cambridge, 2001, 245-258. MR 1842789173

[J1] K. JOHANSSON, Shape fluctuations and random matrices, Comm. Math. Phys. 209 (2000), 437-476. MR 1737991 171, 173, 174

[J2] Discrete orthogonal polynomial ensembles and the Plancherel measure, Ann. of Math. (2) 153 (2001), 259-296. MR 1826414173

[J3] Non-intersecting paths, random tilings and random matrices, Probab. Theory Related Fields 123 (2002), 225 - 280. MR 1900323173

[M] I. G. MACDONALD, Symmetric Functions and Hall Polynomials, 2nd ed., Oxford Math. Monogr., Oxford Univ. Press, Oxford, 1995. MR 1354144 172, 173, 174

[O1] A. OKOUNKOV, Random matrices and random permutations, Internat. Math. Res. Notices 2000, no. 20, 1043 - 1095. MR 1802530173

[O2] - Infinite wedge and random partitions, Selecta Math. (N.S.) 7 (2001), 57 - 81. MR 1856553172

[S] B. E. SAGAN, Shifted tableaux, Schur Q-functions, and a conjecture of R. Stanley, J. Combin. Theory Ser. A 45 (1987) 62 - 103. MR 0883894 173, 174, 179 
[St] R. P. STANLEY, Enumerative Combinatorics, Vol. 2, Cambridge Stud. Adv. Math. 62, Cambridge Univ. Press, Cambridge, 1999. MR 1676282172

[Ste] J. R. STEMBRIDGE, Shifted tableaux and the projective representations of symmetric groups, Adv. Math. 74 (1989), 87 - 134. MR 0991411 173, 174, 180

[TW1] C. A. TRACY and H. WIDOM, Level-spacing distributions and the Airy kernel, Comm. Math. Phys. 159 (1994), 151 - 174. MR 1257246 172, 173

[TW2] - On orthogonal and symplectic matrix ensembles, Comm. Math. Phys. 177 (1996), 727-754. MR 1385083172

[TW3] On the distributions of the lengths of the longest monotone subsequences in random words, Probab. Theory Related Fields 119 (2001), 350-380.

MR 1821139 173, 180

[W] H. WIDOM, On the limit of block Toeplitz determinants, Proc. Amer. Math. Soc. 50 (1975), 167 - 173. MR 0370254186

[Wo] D. WORLEY, A theory of shifted Young tableaux, Ph.D. dissertation, Massachusetts Institute of Technology, Cambridge, 1984, http://theses.mit.edu/Dienst/UI/2.0/Describe/0018.mit.theses/1984-38 173, 174, 179

Tracy

Department of Mathematics, University of California, Davis, Davis, California 95616, USA; tracy@math.ucdavis.edu

\section{Widom}

Department of Mathematics, University of California, Santa Cruz, Santa Cruz, California 95064, USA; widom@math.ucsc.edu 